\title{
Sensitivity to neutrino decay with atmospheric neutrinos at the INO-ICAL detector
}

\author{
Sandhya Choubey, ${ }^{1,2,3, *}$ Srubabati Goswami, ${ }^{4, \dagger}$ Chandan Gupta, ${ }^{3,4, \star}$ S. M. Lakshmi, ${ }^{5, \S}$ and Tarak Thakore ${ }^{6, \|}$ \\ ${ }^{1}$ Harish-Chandra Research Institute, Chhatnag Road, Jhunsi, Allahabad 211 019, India \\ ${ }^{2}$ Department of Physics, School of Engineering Sciences, KTH Royal Institute of Technology, \\ AlbaNova University Center, 10691 Stockholm, Sweden \\ ${ }^{3}$ Homi Bhabha National Institute, Training School Complex, Anushakti Nagar, Mumbai 400085, India \\ ${ }^{4}$ Physical Research Laboratory, Navrangpura, Ahmedabad 380 009, India \\ ${ }^{5}$ Indian Institute of Technology Madras, Chennai 600 036, India \\ ${ }^{6}$ Louisiana State University, Baton Rouge, Louisiana 70803, USA
}

(Received 28 November 2017; published 23 February 2018)

\begin{abstract}
Sensitivity of the magnetized Iron Calorimeter (ICAL) detector at the proposed India-based Neutrino Observatory (INO) to invisible decay of the mass eigenstate $\nu_{3}$ using atmospheric neutrinos is explored. A full three-generation analysis including Earth matter effects is performed in a framework with both decay and oscillations. The wide energy range and baselines offered by atmospheric neutrinos are shown to be excellent for constraining the $\nu_{3}$ lifetime. We find that with an exposure of $500 \mathrm{kton}-\mathrm{yr}$ the ICAL atmospheric experiment could constrain the $\nu_{3}$ lifetime to $\tau_{3} / m_{3}>1.51 \times 10^{-10} \mathrm{~s} / \mathrm{eV}$ at the $90 \%$ C.L. This is 2 orders of magnitude tighter than the bound from MINOS. The effect of invisible decay on the precision measurement of $\theta_{23}$ and $\left|\Delta m_{32}^{2}\right|$ is also studied.
\end{abstract}

DOI: 10.1103/PhysRevD.97.033005

\section{INTRODUCTION}

Neutrino oscillation experiments spanning various energy ranges and baselines have helped in establishing the fact that neutrinos oscillate from one flavor to another. Most of the neutrino oscillation parameters have been pinned down and are now known rather precisely. ${ }^{1}$ The main open questions remaining in neutrino oscillation physics are neutrino mass hierarchy, octant of the mixing angle $\theta_{23}$ and the value of the $C P$ phase $\delta_{C P}$. Several experiments are running or are being planned in order to answer the above-mentioned questions. The leading experimental proposals for the future include the long-baseline experiments DUNE [3] and T2HK [4], reactor experiments

\footnotetext{
sandhya@hri.res.in

†sruba@prl.res.in

*chandang@prl.res.in

${ }^{\S}$ Corresponding author.

ph17ipf06@smail.iitm.ac.in

tarakstar@gmail.com

${ }^{1}$ See $[1,2]$ for the current global best-fit values of the oscillation parameters and the references therein to all the past and on-going experimental efforts.
}

Published by the American Physical Society under the terms of the Creative Commons Attribution 4.0 International license. Further distribution of this work must maintain attribution to the author(s) and the published article's title, journal citation, and DOI. Funded by SCOAP ${ }^{3}$.
JUNO [5] and RENO50 [6], and atmospheric neutrino experiments PINGU [7], ORCA [8] and ICAL $[9,10]$. It is expected that the neutrino oscillation probabilities would change in the presence of new physics. This could be used to constrain new physics scenarios at neutrino oscillation experiments. At the same time, a given new physics scenario could also interfere with the measurement of the standard neutrino oscillation parameters and hence pose a challenge to the proposed experiments, unless ways are found to cancel out their effects through synergistic measurements at multiple experiments. One such new physics scenario is the decay of neutrino during its flight from the source to the detector.

While there is no observational evidence in support for unstable neutrinos, since they are massive, its not unlikely that they would decay. Radiative decays of neutrinos are very severely constrained by cosmological data. Since the measured neutrino masses suggest that the neutrinos would radiatively decay in the microwave energy range, the most stringent bounds are provided by cosmic microwave background data [11], making radiative decay of neutrinos totally uninteresting for neutrino oscillation experiments. However, there still remains the possibility that neutrinos could decay into a lighter fermion state and a beyond standard model boson. The Majoron model [12-14] for instance allows the following decay modes for Majorana neutrinos: $\nu_{i} \rightarrow \nu_{j}+J$ or $\nu_{i} \rightarrow \bar{\nu}_{j}+J$, where $\nu_{j}$ and $\bar{\nu}_{j}$ are lighter neutrino and antineutrino states and $J$ is a Majoron. 
The Majoron in principle could belong to either a singlet or a triplet representation of the standard model gauge group. But the triplet model is severely constrained $[13,14]$ and hence $J$ must predominantly be an electroweak singlet. If the final state fermion is a lighter active neutrino, the decay is called visible decay. On the other hand, if the final state fermion is a sterile state with no standard model interaction, then the decay scenario is termed invisible decay. Even for Dirac neutrinos in extensions of the standard models, one could write down terms in the Lagrangian coupling neutrinos with a light scalar boson and light right-handed neutrinos allowing the decay mode $\nu_{i} \rightarrow \bar{\nu}_{i R}+\chi$, where $\bar{\nu}_{i R}$ is a right-handed singlet neutrino and $\chi$ is an isosinglet scalar carrying lepton number $+2[15,16]$. In this paper, we will work in a scenario where the final state particles remain invisible to the detector.

The lifetime of $\nu_{2}$ (and $\nu_{1}$ ) is constrained by the solar neutrino experiments. Neutrino decay as a solution to the solar neutrino deficit problem was suggested in [17], however, now we know that neutrino decay alone cannot explain this deficit. Attempts to constrain the neutrino lifetime by considering neutrino decay as a subdominant effect along with the leading large mixing angle MikheyevSmirnov-Wolfenstein solution was done in [18-26]. Most of these studies considered the invisible decay scenario. Since $U_{e 3}$ is small, the $\nu_{e}$ state mostly resides in the $\nu_{2}$ and $\nu_{1}$ states and hence all of these studies worked in the two-generation framework. Bounds on the lifetime of $\nu_{2}$ were obtained from a global analysis of solar neutrino data in [24] where the impact of the Sudbury Neutrino Observatory neutral current data was highlighted. It was shown that the bound on $\nu_{2}$ lifetime was $\tau_{2} / m_{2}>8.7 \times 10^{-5} \mathrm{~s} / \mathrm{eV}$ at $99 \%$ C.L. for a three parameter fit. This bound was revisited in [25] (see also [26]) where the authors obtained the 95\% C.L. limit $\tau_{2} / m_{2}>$ $7 \times 10^{-4} \mathrm{~s} / \mathrm{eV}$ for both normal and inverted mass hierarchy and $\tau_{1} / m_{1}>4 \times 10^{-3} \mathrm{~s} / \mathrm{eV}$ for inverted mass hierarchy. These results are very consistent with the earlier analysis of [24] where the 95\% C.L. limit for a one parameter fit is seen to be $\tau_{2} / m_{2}>4.4 \times 10^{-4} \mathrm{~s} / \mathrm{eV}$. The corresponding constraints from SN1987A are stronger [27].

Limits on the lifetime of $\nu_{3}$ come from the atmospheric and long-baseline neutrino experiments. Like in the case of solar neutrinos, any fit with neutrino decay alone [28,29] is unable to explain the atmospheric neutrino zenith angle data. A lot of work has gone into considering decay along with oscillations. The analyses can be broadly classified into two categories depending on the model used. If one considers decay of $\nu_{3}$ to a state with which it oscillates, then the bounds coming from K-decays [30] restrict the corresponding mass squared difference between them to $\Delta m^{2}>0.1 \mathrm{eV}^{2}$ [31]. However, if the state to which $\nu_{3}$ decays is a sterile state then the $\Delta m^{2}$ driving the leading oscillations of $\nu_{\mu}$ is unconstrained. The former case is that of decay to active neutrinos and was studied in the context of atmospheric neutrinos in [31,32] and no good fit was found. The latter is the invisible decay scenario to sterile neutrinos and was analyzed against the atmospheric neutrino data in [33-36]. The invisible decay case can be again classified into two. In one case we can make the assumption that $\Delta m^{2} \ll 10^{-4} \mathrm{eV}^{2}$, causing it to drop out of the oscillation probability. The authors of [34] argued that this could explain the Super-Kamiokande atmospheric neutrino data, however, the Super-Kamiokande collaboration itself reported [35] that this scenario was not supported by their data. The other case of invisible decay is when $\Delta m^{2}$ is left free in the fit to be determined by the data. This case was first proposed by some of us in [33]. The results of [33] were updated in [36] where the authors obtained the limit $\tau_{3} / m_{3}>2.9 \times 10^{-10} \mathrm{~s} / \mathrm{eV}$ for invisible decay at the $90 \%$ C.L. from a combined analysis of Super-Kamiokande atmospheric and MINOS data. More recently, the analysis of oscillation plus invisible decay scenario with unconstrained $\Delta m^{2}$ was performed in [37] in the context of MINOS and T2K data and gave a bound $\tau_{3} / m_{3}>2.8 \times 10^{-12} \mathrm{~s} / \mathrm{eV}$ at $90 \%$ C.L. The constraint for the visible decay scenario using the MINOS and T2K charged as well as neutral current data was performed in [38]. The bounds on neutrino lifetime could be improved considerably by observations at IceCube using cosmological baselines [39-42].

All the above-mentioned papers which considered neutrino decay alongside oscillations performed their analysis in the framework of two generations and did not take Earth matter effects into account. Recently a three-generation analysis including Earth matter effect and decay in the context of the Deep Underground Neutrino Experiment (DUNE) was performed in [43] for visible decays and [44] for invisible decays. It was shown that DUNE could improve the bound on $\tau_{3} / m_{3}$ for the invisible decay case by at least an order of magnitude compared to the current limits from MINOS and T2K. In this work, we consider invisible neutrino decay within a three-generation oscillation framework in the context of atmospheric neutrinos and include Earth matter effects. Atmospheric neutrinos span many orders of magnitude in energy and baseline. Since the effect of neutrino decay increases for lower energies and longer baselines, atmospheric neutrino experiments are expected to give a tighter bound on $\tau_{3} / m_{3}$ than the proposed longbaseline experiments. We will study the sensitivity of the atmospheric neutrinos at INO to neutrino decay.

The India-based Neutrino Observatory (INO) is a proposed underground laboratory in India, which plans to house a 50 kton magnetized Iron Calorimeter (ICAL). The detector will be mainly sensitive to muon-type neutrinos, which are detected through the observation of a muon track and the accompanying hadron shower in a charged current interaction. The detector response to muons [45-48] and hadrons [49-52] has been performed via the GEANT4-based [53-55] detector simulation code for ICAL. This detector owing to its magnetization can distinguish between neutrino and antineutrino events which makes it an excellent 
detector to determine the neutrino mass hierarchy [9,56-60]. ICAL will also perform precision measurements of $\left|\Delta m_{32}^{2}\right|$ and the mixing angle $\theta_{23}[9,58,59,61-64]$. In addition, there are a variety of new physics scenarios which could be constrained and/or discovered at ICAL. Some of the new physics scenarios studied by the INO collaboration include $C P T$ violation [65], dark matter [66], nonstandard neutrino interactions [67] and sterile neutrino oscillations [68]. In this work we will study in detail the sensitivity of ICAL to invisible neutrino decay using the full physics analysis simulation framework of ICAL. We will also study the effect of invisible neutrino decay on the precision measurement of $\left|\Delta m_{32}^{2}\right|$ and the mixing angle $\theta_{23}$.

The paper is organized as follows. The scenario of invisible decay plus oscillations for three-generation mixing and oscillations in Earth matter are discussed in Sec. II.

$$
U=\left(\begin{array}{c}
c_{12} c_{13} \\
-c_{23} s_{12}-s_{23} s_{13} c_{12} e^{i \delta} \\
s_{23} s_{12}-c_{23} s_{13} c_{12} e^{i \delta}
\end{array}\right.
$$

where $c_{i j}=\cos \theta_{i j}, s_{i j}=\sin \theta_{i j} ; \theta_{i j}$ are the mixing angles and $\delta$ is the $C P$ violating phase.

The mass of $\nu_{s}$ is such that when the hierarchy is normal, $m_{s}<m_{1}<m_{2}<m_{3}$. Since $\nu_{s}$ does not mix with the active neutrinos, the propagation equation is not affected by this. The effect of decay is included in the three-flavor evolution equation in the presence of Earth matter as follows:

$$
\begin{aligned}
i \frac{d \tilde{\nu}}{d t} & =\frac{1}{2 E}\left[U \mathbb{M}^{2} U^{\dagger}+\mathbb{A}_{C C}\right] \tilde{\nu}, \\
\mathbb{M}^{2} & =\left(\begin{array}{ccc}
0 & 0 & 0 \\
0 & \Delta m_{21}^{2} & 0 \\
0 & 0 & \Delta m_{31}^{2}-i \alpha_{3}
\end{array}\right), \\
\text { and } \quad \mathbb{A}_{C C} & =\left(\begin{array}{ccc}
A_{c c} & 0 & 0 \\
0 & 0 & 0 \\
0 & 0 & 0
\end{array}\right),
\end{aligned}
$$

where $E$ is the neutrino energy, $\alpha_{3}=m_{3} / \tau_{3}$ is the decay constant in units of $\mathrm{eV}^{2}, m_{3}$ is the mass of $\nu_{3}$ and $\tau_{3}$ its rest frame lifetime. Since the term $\alpha_{3}$ appears in the propagation equation along with $\Delta m_{31}^{2}$, it has to be in units of $\mathrm{eV}^{2}$. The conversion factor used here is $1 \mathrm{eV} / \mathrm{s}=6.58 \times 10^{-16} \mathrm{eV}^{2}$. The matter potential is

$$
\begin{aligned}
A_{c c} & =2 \sqrt{2} G_{F} n_{e} E \\
& =7.63 \times 10^{-5} \mathrm{eV}^{2} \rho(g m / c c) E(\mathrm{GeV}),
\end{aligned}
$$

where $G_{F}$ is the Fermi constant and $n_{e}$ is the electron number density in matter and $\rho$ is the matter density. For
The simulation of events and $\chi^{2}$ analysis are explained in Sec. III. In Sec. IV we present our results for the sensitivity to the decay parameter $\tau_{3} / m_{3}$. The effects of the presence of decay on the precision measurements of $\sin ^{2} \theta_{23}$ and $\left|\Delta m_{32}^{2}\right|$ are discussed in Secs. VA and V B, respectively. The exclusion contours are presented in Sec. VC. Conclusions are presented in Sec. VI.

\section{INVISIBLE DECAY AND OSCILLATIONS IN THE PRESENCE OF MATTER}

In this section we consider the oscillations and decay of $\nu_{3}$ in the presence of matter. Let the state $\nu_{3}$ decay invisibly via $\nu_{3} \rightarrow \nu_{s}+J$, where $J$ is a pseudoscalar and $\nu_{s}$ is a sterile neutrino. Since $\nu_{s}$ does not mix with the three active neutrinos, the mixing matrix $U$ in vacuum [69-71] is given by

$$
\left.\begin{array}{cc}
s_{12} c_{13} & s_{13} e^{-i \delta} \\
c_{23} c_{12}-s_{23} s_{13} s_{12} e^{i \delta} & s_{23} c_{13} \\
-s_{23} c_{12}-c_{23} s_{13} s_{12} e^{i \delta} & c_{23} c_{13}
\end{array}\right),
$$

antineutrinos, both the sign of $A_{c c}$ and the phase $\delta$ in Eq. (2) are reversed.

\section{A. Effect of the decay term}

The decay term is of the form of $\exp (-\alpha L / E)$. No decay corresponds to $\alpha=0$ and the exponential term as 1 whereas complete decay will be when the exponential term tends to 0 . The effect of the decay parameter $\alpha$ for various $L / E$ values can be understood from Fig. 1 in which $\exp (-\alpha L / E)$ vs $L / E$ is plotted for the values $\alpha=10^{-3}$, $10^{-4}, 10^{-5}$ and $10^{-6} \mathrm{eV}^{2}$. This figure gives an indication towards what are the values of $\alpha$ to which a given experiment spanning a specified $L / E$ range can be sensitive to. For instance, the red shaded region in Fig. 1 indicates the $L / E$ range covered by the narrow band $\mathrm{NO} \nu \mathrm{A}$ neutrino beam $(E=1-3 \mathrm{GeV})$. It can be seen from the figure that $\mathrm{NO} \nu$ A's sensitivity is limited to larger values of $\alpha$; i.e $10^{-3}$ and $10^{-4} \mathrm{eV}^{2}$ for which the exponential terms show substantial departure from the no decay value of 1 . The blue shaded region corresponds to the baseline $L=$ $9700 \mathrm{~km}$ with $E=0.5-25 \mathrm{GeV}$, respectively. These are the typical values for an atmospheric neutrino experiment. This range of $L / E$ is sensitive to a wider range of $\alpha$ from $\sim 10^{-6}-10^{-3} \mathrm{eV}^{2}$ owing to the fact that it covers more $L / E$.

The ranges of $\exp (-\alpha L / E)$ values for various values of $\alpha$ accessible for the specified range of $L / E$ for a given baseline are shown in Table I.

For a given $L$, a broader range of $E$ will improve the sensitivity to $\alpha$; on the other hand, for a given $E$ range the sensitivity to $\alpha$ will increase if longer baselines are available. In principle any experiment which spans over 


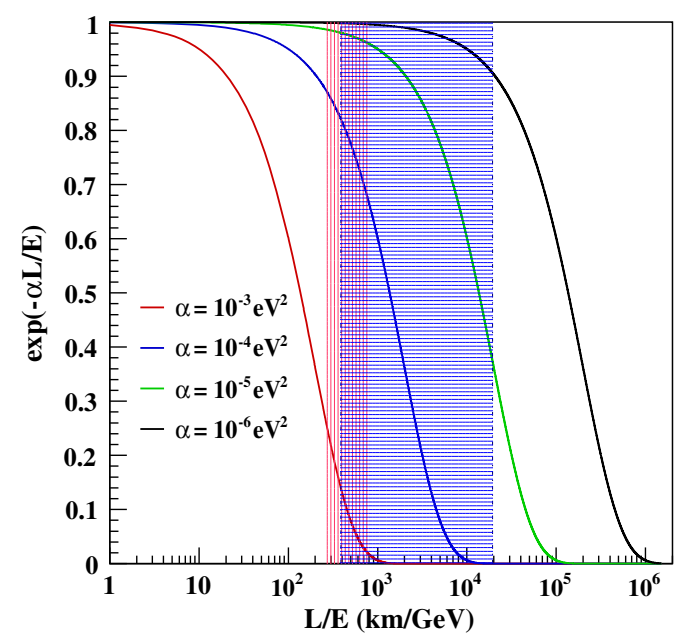

FIG. 1. The value of $\exp (-\alpha L / E)$ as a function of $L / E$ for different values of the decay parameter $\alpha$. The red shaded region denotes the $L / E$ range accessible with $\mathrm{NO} \nu \mathrm{A}$ narrow band neutrino beam $(E=1-3 \mathrm{GeV})$ the dashed blue shaded region indicates the range for $L=9700 \mathrm{~km}$, when $E$ is in the range $0.5-25 \mathrm{GeV}$.

a wide range of $L / E$ will have a better sensitivity to decay; with larger $L / E$ s being sensitive to smaller values of $\alpha$ and vice versa. Atmospheric neutrino oscillation experiments fulfill this exact requirement. If we consider the neutrino energy range of $0.5-25 \mathrm{GeV}$, atmospheric neutrinos will span the $L / E$ range of $[0.6,25484](\mathrm{km} / \mathrm{GeV})$ which includes all possible baselines from $15 \mathrm{~km}$ to the Earth's diameter. The INO ICAL detector becomes relevant in this context. Since ICAL can detect neutrinos in the range $0.5-25 \mathrm{GeV}$ [59] and since it is an atmospheric neutrino experiment, it will be sensitive to a wide range of $\alpha$ values. As seen from Fig. 1 ICAL should give a sensitivity to $\alpha=10^{-6} \mathrm{eV}^{2}$ also. The sensitivity to low $\alpha$ values comes from the low energy part of the spectrum, while the higher

TABLE I. Allowed ranges of $L / E$ in $\mathrm{km} / \mathrm{GeV}$ for two fixed baselines 810 and $9700 \mathrm{~km}$ with detectable neutrino energies as 1-3 GeV and $0.5-25 \mathrm{GeV}$, respectively. The maximum and minimum values of $\exp (-\alpha L / E)$ for various $\alpha$ values for these $L / E$ s are also shown.

\begin{tabular}{lccccc}
\hline \hline $\begin{array}{l}L \\
(\mathrm{~km})\end{array}$ & $\begin{array}{c}L / E(\mathrm{~min}) \\
(\mathrm{km} / \mathrm{GeV})\end{array}$ & $\begin{array}{c}L / E(\max ) \\
(\mathrm{km} / \mathrm{GeV})\end{array}$ & $\begin{array}{c}\alpha \\
\left(\mathrm{eV}^{2}\right)\end{array}$ & $\begin{array}{c}\exp (-\alpha L / E) \\
(\min )\end{array}$ & $\begin{array}{c}\exp (-\alpha L / E) \\
(\max )\end{array}$ \\
\hline 810 & 270 & 810 & $10^{-3}$ & 0.016 & 0.254 \\
& & & $10^{-4}$ & 0.663 & 0.872 \\
& & & $10^{-5}$ & 0.959 & 0.986 \\
& & & $10^{-6}$ & 0.996 & 0.998 \\
9700 & \multirow{2}{*}{388} & \multirow{2}{*}{19400} & $10^{-3}$ & 0 & 0.14 \\
& & & $10^{-4}$ & 0 & 0.82 \\
& & & $10^{-5}$ & 0.37 & 0.98 \\
& & & $10^{-6}$ & 0.91 & 1 \\
\hline \hline
\end{tabular}

energy parts of the spectrum will help us rule out larger values of $\alpha$.

\section{B. Full three-flavor oscillations with decay in Earth matter}

We perform an exact numerical calculation of the neutrino oscillation probabilities within the framework of three-generation mixing and invisible decay of $\nu_{3}$. The oscillation probabilities are computed in the presence of Earth matter assuming the Preliminary Reference Earth Model (PREM) density profile [72]. The oscillation probabilities $P_{\mu \mu}, P_{e \mu}, \bar{P}_{\mu \mu}$ and $\bar{P}_{e \mu}$ as a function of neutrino energy for the baseline $L=9700 \mathrm{~km}$, for various values of the decay parameter $\alpha_{3}$ and $\theta_{23}$ are shown in Fig. 2. The following values have been used to generate these:

(1) $\delta_{C P}=0^{\circ}$

(2) $\theta_{12}=34.08^{\circ} ; \theta_{23}=39^{\circ}, 45^{\circ}, 51^{\circ} ; \theta_{13}=8.5^{\circ}$

(3) $\Delta m_{21}^{2}=7.6 \times 10^{-5}\left(\mathrm{eV}^{2}\right) ;\left|\Delta m_{32}^{2}\right|=2.4 \times 10^{-3}\left(\mathrm{eV}^{2}\right)$

(4) $\alpha_{3}=0,10^{-4}, 10^{-3}\left(\mathrm{eV}^{2}\right)$.

First let us consider the effect of $\alpha_{3}$ alone for a given $\theta_{23}$. The plots for $\alpha_{3}=0$ correspond to the oscillation only case and as the value of $\alpha_{3}$ increases the effect of decay becomes prominent which can be seen from the figure. In general the effect of decay is seen to be more for the lower energy neutrinos. For the decay constant $\alpha_{3}=10^{-4} \mathrm{eV}^{2}$, the effect of decay increases and the neutrino probabilities show significant depletion as compared to the no decay case for neutrino energies up to $\sim 15 \mathrm{GeV}$. The presence of decay reduces the oscillation amplitude near maxima and elevates it near minima. As $\alpha_{3}$ increases to $10^{-3} \mathrm{eV}^{2}$, the survival probabilities of the neutrinos and antineutrinos show a difference over the entire energy range considered. We also note that the effect of decay is mainly to damp out the oscillatory behavior in the probabilities. For the large decay case the oscillatory behavior is seen to be largely washed out. From Fig. 2 it can be seen that the relative change in the oscillation probability due to decay is more for $\bar{P}_{\mu \mu}$ than $P_{\mu \mu}$ whereas the relative change in $P_{e \mu}$ is more compared to that in $\bar{P}_{e \mu}$. Hence, the contribution to the $\alpha_{3}$ sensitivity $\chi^{2}$ will be more from antineutrino events in the former case and neutrino events in the latter case. However, since $P_{\mu \mu}$ and $\bar{P}_{\mu \mu}$ are the dominant channels at ICAL, the major contribution to $\alpha_{3}$ sensitivity is expected to come from antineutrino events in the present study.

Now let us look at the effect of $\theta_{23}$ alone for a given $\alpha_{3}$ value. The effect of $\theta_{23}$ is also to vary the oscillation amplitude. In general, $P_{\mu \mu}$ and $\bar{P}_{\mu \mu}$ decrease with increase in $\theta_{23}$. However beyond $13 \mathrm{GeV}$, for $\alpha_{3}=0$ and $10^{-4} \mathrm{eV}^{2}$, $\theta_{23}=45^{\circ}$ gives the lowest probability compared to those for $39^{\circ}$ and $51^{\circ}$, though the relative variation is much less. From the plots in the lower panels of Fig. 2 we see that $P_{e \mu}$ and $\bar{P}_{e \mu}$ increase with $\theta_{23}$, the increase in $P_{e \mu}$ is 

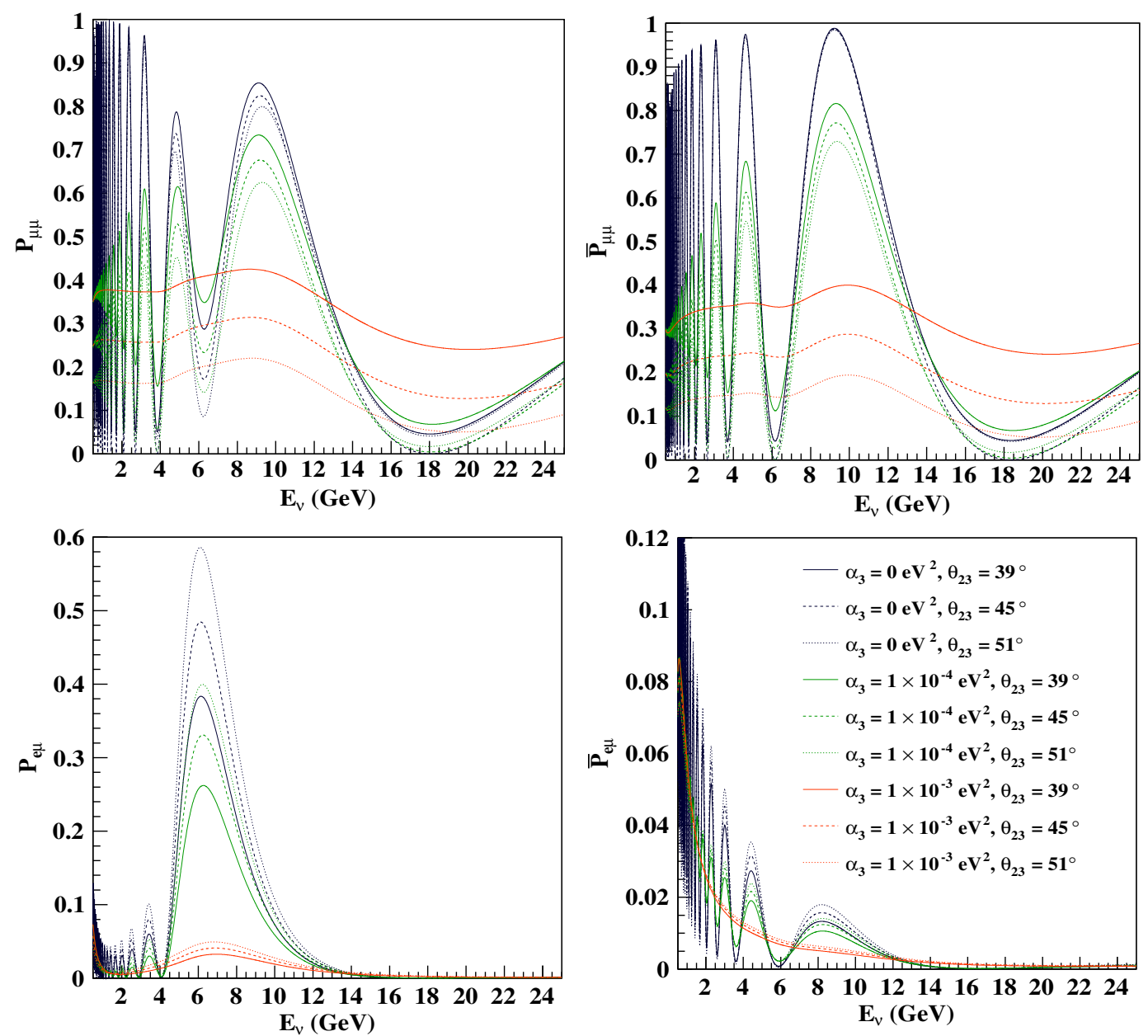

FIG. 2. Oscillation probabilities in matter for $\alpha_{3}=0,1 \times 10^{-4}$ and $1 \times 10^{-3} \mathrm{eV}^{2}$ and $\theta_{23}=39^{\circ}, 45^{\circ}$ and $51^{\circ}$, for the baseline $L=9700 \mathrm{~km}$ in the energy range $E_{\nu}=0.5-25 \mathrm{GeV}$. (Top-left) $P_{\mu \mu}$ and (top-right) $\bar{P}_{\mu \mu}$; (bottom-left) $P_{e \mu}$ and (bottom-right) $\bar{P}_{e \mu}$. NH is taken as the true hierarchy.

larger than that in $\bar{P}_{e \mu}$. For all values of $\theta_{23}, P_{e \mu}$ and $\bar{P}_{e \mu}$ decrease.

Since both $\alpha_{3}$ and $\theta_{23}$ affect the oscillation amplitudes, when combined in the following way, similar probabilities can be obtained. The combination of $\theta_{23}$ in the first octant + a larger (smaller) value of $\alpha_{3}$ will give a probability similar to that with $\theta_{23}$ in the second octant + a smaller (larger) value of $\alpha_{3}$ for $P_{\mu \mu}$ and $\bar{P}_{\mu \mu}\left(P_{e \mu}\right.$ and $\left.\bar{P}_{e \mu}\right)$. Since the event spectrum is dominated by $P_{\mu \mu}$ and $\bar{P}_{\mu \mu}$ events, this combined effect will affect the sensitivity/discovery potential to/of $\alpha_{3}$ and the precision measurement on $\theta_{23}$, which is discussed in Sec. V.

\section{DETAILS OF NUMERICAL SIMULATIONS}

ICAL will be a 50 kton magnetized iron detector which is optimized for the detection of atmospheric $\nu_{\mu}$ and $\bar{\nu}_{\mu}$. Both $\nu_{\mu}\left(\bar{\nu}_{\mu}\right)$ and $\nu_{e}\left(\bar{\nu}_{e}\right)$ fluxes can contribute to the $\nu_{\mu}\left(\bar{\nu}_{\mu}\right)$ events observed at ICAL. Hence the number of events detected by ICAL will be

$$
\begin{aligned}
\frac{d^{2} N}{d E_{\mu} d \cos \theta_{\mu}}= & t \times n_{d} \times \int d E_{\nu} d \cos \theta_{\nu} d \phi_{\nu} \\
& \times\left[P_{\mu \mu}^{m} \frac{d^{3} \Phi_{\mu}}{d E_{\nu} d \cos \theta_{\nu} d \phi_{\nu}}+P_{e \mu}^{m} \frac{d^{3} \Phi_{e}}{d E_{\nu} d \cos \theta_{\nu} d \phi_{\nu}}\right] \\
& \times \frac{d \sigma_{\mu}\left(E_{\nu}\right)}{d E_{\mu} d \cos \theta_{\mu}}
\end{aligned}
$$

where $n_{d}$ is the number of nucleon targets in the detector, $\sigma_{\mu}$ is the differential neutrino interaction cross section in terms of the energy and direction of the muon produced, $\Phi_{\mu}$ and $\Phi_{e}$ are the $\nu_{\mu}$ and $\nu_{e}$ fluxes and $P_{\alpha \beta}^{m}$ is the oscillation probability of $\nu_{\alpha} \rightarrow \nu_{\beta}$ in matter and in the presence of decay. A sample of 1000 years of unoscillated neutrino events is generated using NUANCE-3.5 neutrino generator [73], in which the Honda 3D atmospheric neutrino fluxes [74] along with neutrino-nucleus cross sections and a simplified ICAL detector geometry are incorporated. Each event is oscillated by multiplying with the relevant 
oscillation probability including decay and oscillations in Earth matter assuming PREM density profile [72]. The probabilities are obtained by solving the propagation equation in matter in the presence of decay. The events are then smeared according to the resolutions and efficiencies obtained from $[45,46]$. These two steps are done on an event by event basis for the entire 1000 year sample. Both "data" and theory are generated via this method, data with the central values of the parameters as described in Table II and theory by varying them in their respective $3 \sigma$ ranges. Afterwards the oscillated samples of 1000 years of events, both data and theory are scaled down to the required number of years, ten for our current analysis. This is done to reduce the effect of Monte Carlo fluctuations on sensitivity studies.

In the current analysis, the efficiencies and resolutions of muons in the central region of the detector $[45,46]$ have been used over the entire detector. These resolutions and efficiencies have been obtained by the INO collaboration via detailed detector simulations using a GEANT4-based simulation toolkit for ICAL. The central region of the ICAL detector $[45,46]$ has the best efficiencies and resolutions for muons, the few-GeV muons in ICAL have a momentum resolution of $\sim 10 \%$ and direction resolution of $\sim 1^{\circ}$ on the average. Their relative charge identification efficiencies is about $\sim 99 \%$. However, ICAL has two more regions, namely the peripheral $[47,48]$ and side regions depending on the magnitude and strength of the magnetic field. The peripheral region which has lesser reconstruction efficiencies but only slightly worse resolutions compared to the central region, constitutes $50 \%$ of the detector. Hence, in a realistic scenario where the efficiencies and resolutions in different regions are taken appropriately, the results obtained with ten years of running of 50 kton of ICAL will only be obtained by increasing the run time to $\sim 11.3$ years, as mentioned in [59].

Since the charged current $\nu_{\mu}\left(\bar{\nu}_{\mu}\right)$ interactions have $\mu^{-}\left(\mu^{+}\right)$in the final state along with the hadron shower, and since ICAL is capable of measuring the energy of the hadron shower, we include in our analysis the data on those as well. It was reported in [49] from ICAL simulations that hadrons in ICAL have energy resolutions of $85 \%$ at $1 \mathrm{GeV}$ and $36 \%$ at $15 \mathrm{GeV}$ and the events are smeared accordingly before including them in the final 3D-binned analysis which includes muons binned in observed energy and direction and hadrons binned in energy. There are 15 bins in $E_{\mu}^{\mathrm{obs}}$ between $(0.5-25) \mathrm{GeV}, 21$ bins in $\cos \theta_{\mu}^{\mathrm{obs}}$ between $(-1,+1)$ and 4 bins in $E_{\text {had }}^{\text {obs }}$ between $(0-15) \mathrm{GeV}$, thus giving 1260 bins. More details of the binning scheme and the numerical simulations can be found in Ref. [59].

The true values and the $3 \sigma$ ranges of the oscillation parameters used to generate the probabilities are given in Table II. Since ICAL is not directly sensitive to $\delta_{C P}$, it is taken as $0^{\circ}$ in this analysis and kept fixed. The 1-2 oscillation parameters $\Delta m_{21}^{2}$ and $\sin ^{2} \theta_{12}$ are also kept
TABLE II. Oscillation parameters used in this analysis. For fixed parameter studies all parameters are kept at their true values. While applying marginalization, only the parameter for which the sensitivity study is being performed is kept fixed and the others are varied in their respective $3 \sigma$ ranges.

\begin{tabular}{lcc}
\hline \hline Parameter & True value & Marginalization range \\
\hline$\theta_{13}$ & $8.5^{\circ}$ & {$\left[7.80^{\circ}, 9.11^{\circ}\right]$} \\
$\sin ^{2} \theta_{23}$ & 0.5 & {$[0.39,0.64]$} \\
$\Delta m_{32}^{2}$ & $2.366 \times 10^{-3} \mathrm{eV}^{2}$ & {$[2.3,2.6] \times 10^{-3} \mathrm{eV}^{2}(\mathrm{NH})$} \\
$\sin ^{2} \theta_{12}$ & 0.304 & Not marginalized \\
$\Delta m_{21}^{2}$ & $7.6 \times 10^{-5} \mathrm{eV}^{2}$ & Not marginalized \\
$\delta_{C P}$ & $0^{\circ}$ & Not marginalized \\
\hline \hline
\end{tabular}

fixed throughout our analysis. For the remaining parameters two types of analyses are performed-one with fixed parameter and the other with marginalization. In the former all parameters are kept fixed, while in the latter the parameters other than the one for which the sensitivity study is done are marginalized in their respective $3 \sigma$ ranges shown in Table II.

To statistically analyze the data, we define the following $\chi^{2}$ function:

$$
\begin{aligned}
\chi^{2}= & \xi_{l}^{ \pm}, \xi_{6} \sum_{i=1}^{N_{E_{\mu}^{\text {obs }}}} N_{j=1}^{N_{\text {cos } \theta_{\mu b}^{\text {obs }}}} \sum_{k=1}^{N_{E^{\prime \text { obs }}}} 2\left[\left(T_{i j k}^{+}-D_{i j k}^{+}\right)-D_{i j k}^{+} \ln \left(\frac{T_{i j k}^{+}}{D_{i j k}^{+}}\right)\right] \\
& +2\left[\left(T_{i j k}^{-}-D_{i j k}^{-}\right)-D_{i j k}^{-} \ln \left(\frac{T_{i j k}^{-}}{D_{i j k}^{-}}\right)\right] \\
& +\sum_{l^{+}=1}^{5} \xi_{l^{+}}^{2}+\sum_{l^{-}=1}^{5} \xi_{l^{-}}^{2}+\xi_{6}^{2}
\end{aligned}
$$

Here $i, j, k$ sum over muon energy, muon angle and hadron energy bins, respectively. The number of predicted (theory) events with systematic errors in each bin are given by

$$
\begin{aligned}
& T_{i j k}^{+}=T_{i j k}^{0+}\left(1+\sum_{l^{+}=1}^{5} \pi_{i j k}^{l^{+}} \xi_{l^{+}}+\pi_{6} \xi_{6}\right) ; \\
& T_{i j k}^{-}=T_{i j k}^{0-}\left(1+\sum_{l^{-}=1}^{5} \pi_{i j k}^{l^{-}} \xi_{l^{-}}-\pi_{6} \xi_{6}\right) .
\end{aligned}
$$

The number of theory events without systematic errors in a bin is given by $T_{i j k}^{0 \pm}$ and the observed events (data) per bin are given by $D_{i j k}^{ \pm}$. It should be noted that both $D_{i j k}^{ \pm}$and $T_{i j k}^{0 \pm}$ are obtained from the scaled NUANCE neutrino events as mentioned earlier. The following values are taken for the systematic uncertainties [75,76]: $\pi_{1}=20 \%$ flux normalization error, $\pi_{2}=10 \%$ cross section error, $\pi_{3}=5 \%$ tilt error, $\pi_{4}=5 \%$ zenith angle error, $\pi_{5}=5 \%$ overall systematics and $\pi_{6}=2.5 \%$ on $\Phi_{\nu_{\mu}} / \Phi_{\bar{\nu}_{\mu}}$ ratio. These are included in the analysis via the pull method. The "tilt" 
error is incorporated as follows. The event spectrum with the predicted values of atmospheric neutrino fluxes is calculated and then shifted according to the relation:

$$
\Phi_{\delta}(E)=\Phi_{0}(E)\left(\frac{E}{E_{0}}\right)^{\delta} \simeq \Phi_{0}(E)\left(1+\delta \ln \frac{E}{E_{0}}\right)
$$

where $E_{0}$ is $2 \mathrm{GeV}$, and $\delta$ is the $1 \sigma$ systematic tilt error $(5 \%)$. Flux error is included as the difference $\Phi_{\delta}(E)-\Phi_{0}(E)$.

A prior of $8 \%$ at $1 \sigma$ is added to $\sin ^{2} 2 \theta_{13}$. This is the only prior in this calculation. No prior is imposed at all on the quantities whose sensitivities are to be studied, i.e on $\alpha_{3}$, $\theta_{23}$ and $\left|\Delta m_{32}^{2}\right|$. The contribution from prior to the $\chi^{2}$ is

$$
\chi_{\text {prior }}^{2}=\left(\frac{\sin ^{2} 2 \theta_{13}-\sin ^{2} 2 \theta_{13}^{\text {true }}}{\sigma\left(\sin ^{2} 2 \theta_{13}\right)}\right)^{2}
$$
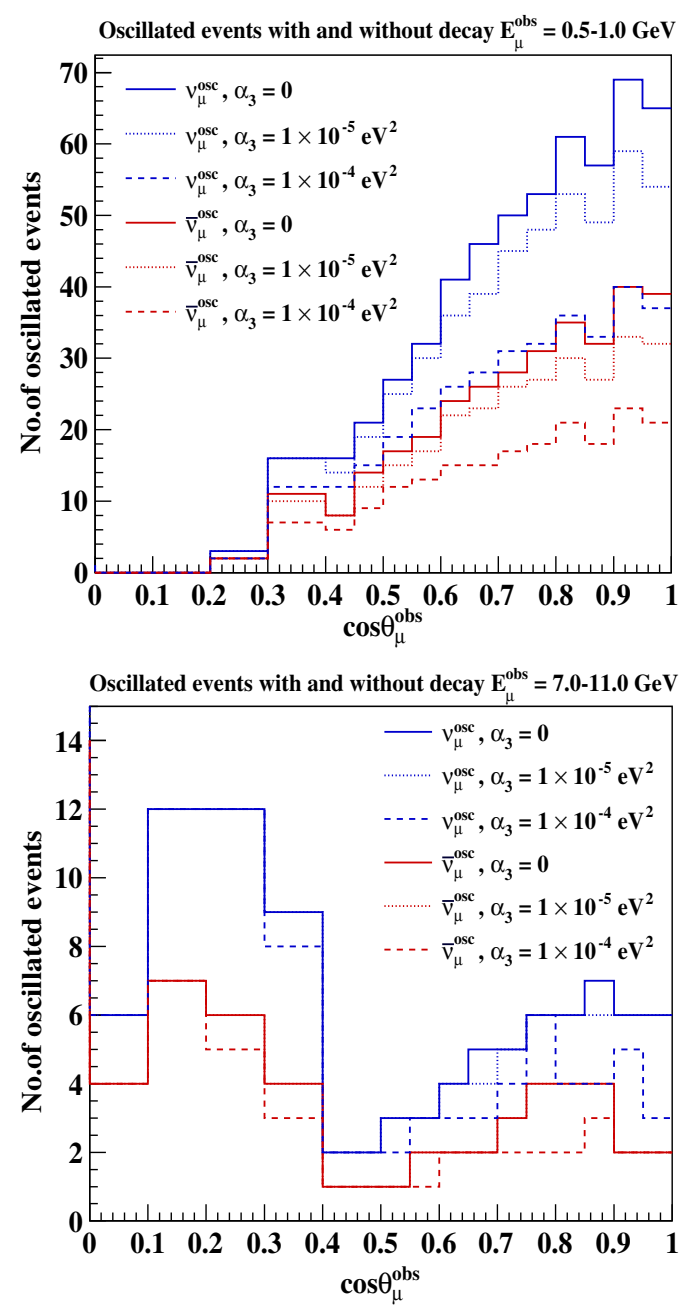

where $\sigma\left(\sin ^{2} 2 \theta_{13}\right)=0.08 \times \sin ^{2} 2 \theta_{13}^{\text {true }}$. Hence, the final $\chi^{2}$ for ICAL will be

$$
\chi_{\mathrm{ICAL}}^{2}=\chi^{2}+\chi_{\text {prior }}^{2},
$$

where $\chi^{2}$ is given by Eq. (6).

\section{SENSITIVITY OF ICAL TO $\alpha_{3}$}

The results of the sensitivity studies of ICAL to $\alpha_{3}$ are presented in this section. We first show how the number of oscillated events change with decay as a function of zenith angle and energy. Then we proceed further to discuss the sensitivity as well as the discovery potential of ICAL to neutrino decay and the bound on $\alpha_{3}$ from our analysis.

\section{A. Effect of decay on the number of oscillated events}

In Fig. 3, we show the zenith angle distribution of the $\nu_{\mu}$ and $\bar{\nu}_{\mu}$ events for different values of the decay constant $\alpha_{3}$.
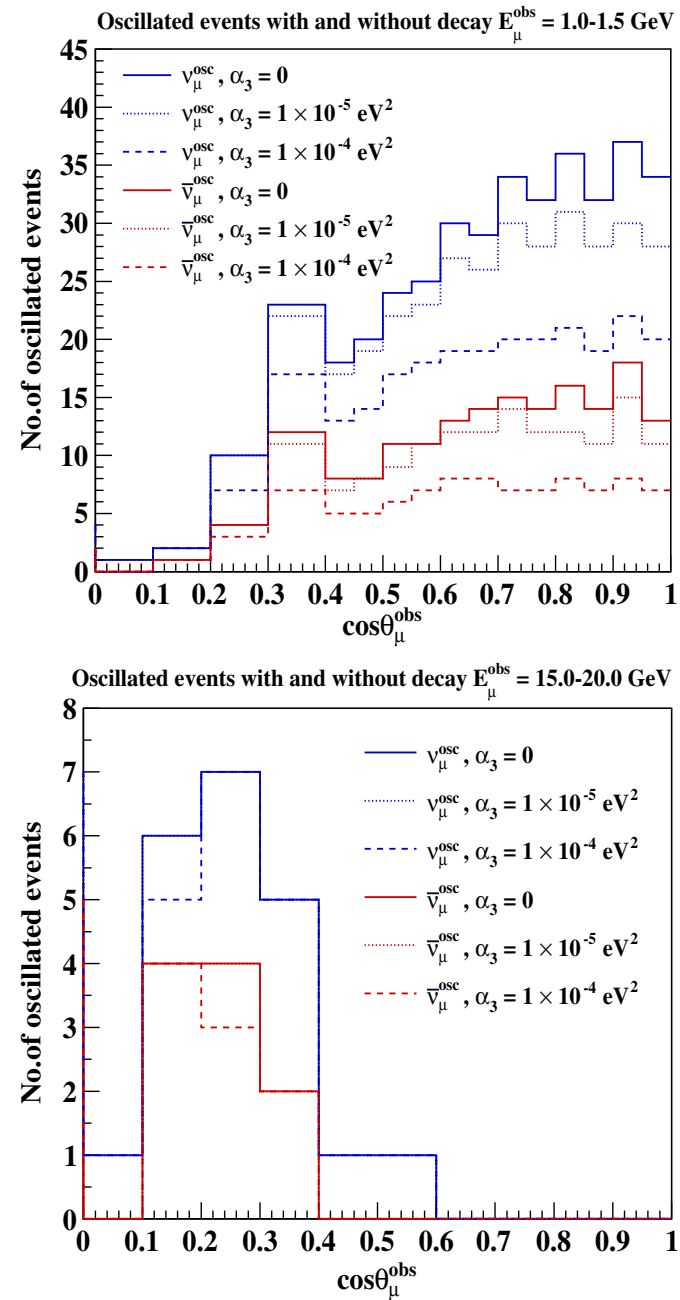

FIG. 3. Oscillated $\nu_{\mu}$ and $\bar{\nu}_{\mu}$ events for each $E_{\mu}^{\mathrm{obs}}$ bin as a function of $\cos \theta_{\mu}^{\mathrm{obs}}$ for $\alpha_{3}=0,1 \times 10^{-5}$ and $1 \times 10^{-4} \mathrm{eV}^{2}$. The other parameters are set to their central values as in Table II. It should be noted that the y-axes are not the same. Only up-coming events (oscillated) are shown here. 

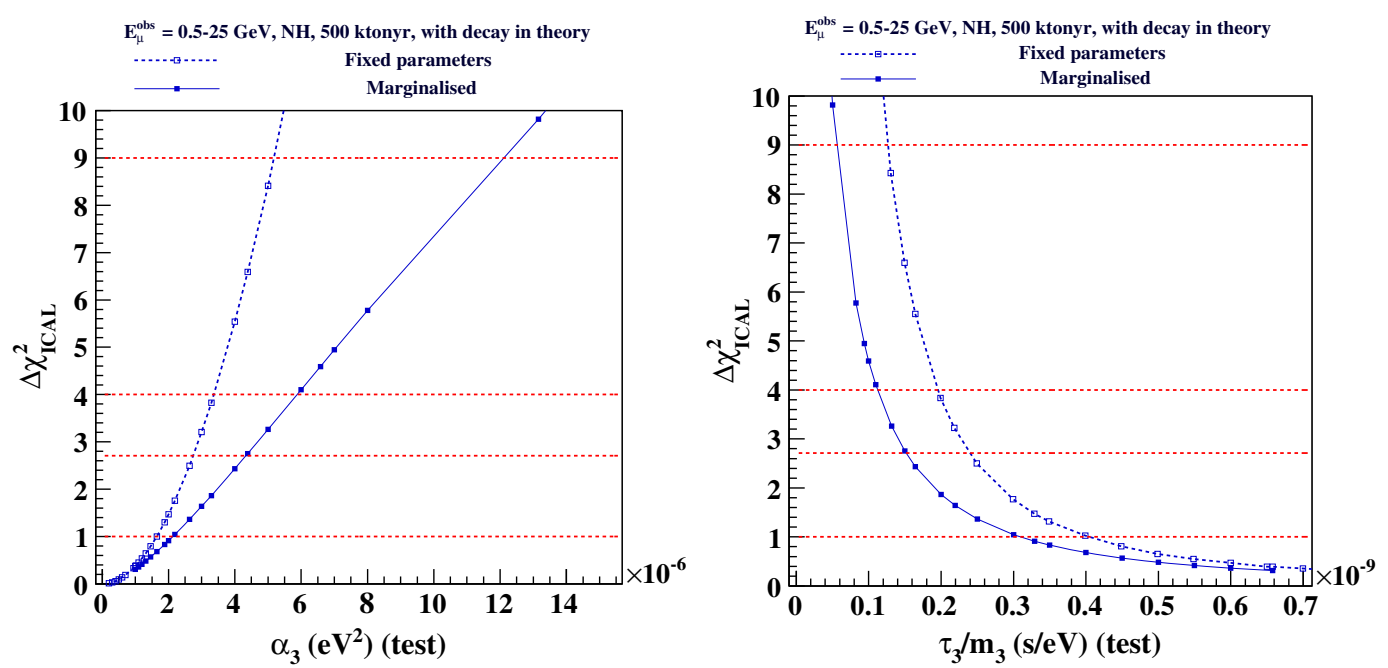

FIG. 4. Expected sensitivity of ICAL to neutrino decay. The expected $\chi^{2}$ is shown as a function of $\alpha_{3}$ (test) $\mathrm{eV}^{2}$ (left panel) and $\tau_{3} / m_{3}$ (test) (s/eV) (right panel) with $500 \mathrm{kton}-\mathrm{yr}$ exposure of ICAL.

The four panels are for four different energy bins. The convention used in these plots is such that $\cos \theta_{\mu}^{\mathrm{obs}}=[0,1]$ indicates the up-coming neutrinos. It can be seen from the figure that both $\nu_{\mu}$ and $\bar{\nu}_{\mu}$ events deplete with an increase in

TABLE III. Sensitivity to $\alpha_{3}\left(\mathrm{eV}^{2}\right)$ and $\tau_{3} / m_{3}(\mathrm{~s} / \mathrm{eV})$ with 500 kton year exposure of ICAL assuming $\mathrm{NH}$ as the true hierarchy.

\begin{tabular}{llcl}
\hline \hline Analysis type & $\chi^{2}$ & $\alpha_{3}\left(\mathrm{eV}^{2}\right)$ & $\tau_{3} / m_{3}(\mathrm{~s} / \mathrm{eV})$ \\
\hline Fixed parameters & 1 & $1.65 \times 10^{-6}$ & $3.99 \times 10^{-10}$ \\
& 2.71 & $2.73 \times 10^{-6}$ & $2.39 \times 10^{-10}$ \\
& 4 & $3.37 \times 10^{-6}$ & $1.96 \times 10^{-10}$ \\
& 9 & $5.19 \times 10^{-6}$ & $1.28 \times 10^{-10}$ \\
Marginalized & 1 & $2.13 \times 10^{-6}$ & $3.03 \times 10^{-10}$ \\
& 2.71 & $4.36 \times 10^{-6}$ & $1.51 \times 10^{-10}$ \\
& 4 & $5.89 \times 10^{-6}$ & $1.12 \times 10^{-10}$ \\
& 9 & $1.21 \times 10^{-5}$ & $5.66 \times 10^{-11}$ \\
\hline \hline
\end{tabular}

the value of $\alpha_{3}$. We also note that the effect of decay is more prominent in the lower energy bins. With increase in energy, there is no significant effect of decay on the number of events if the decay parameter is less than $10^{-4} \mathrm{eV}^{2}$ as can be seen from the lower panels.

\section{B. Sensitivity to the decay parameter $\alpha_{3}$}

In this section, first the study of the sensitivity of ICAL to $\alpha_{3}$ is presented with 500 kton $-\mathrm{yr}$ exposure of the detector taking normal hierarchy $(\mathrm{NH})$ as the true hierarchy. To that end, we simulate the prospective data for no decay and fit it with a theory of oscillation plus decay. The corresponding $\chi^{2}$ is shown as a function of $\alpha_{3}$ (test) in the left panel of Fig. 4.

The blue dashed curve is obtained for a fixed parameter fit while the blue solid one corresponds to the sensitivity when the $\chi^{2}$ is marginalized over all oscillation parameters as described in Sec. III. A comparison of the solid and

TABLE IV. Limits on the decay parameter from various experiments and neutrino sources spanning different $L / E$.

\begin{tabular}{lcc}
\hline \hline Experiment/source & $\tau / m(\mathrm{~s} / \mathrm{eV})(90 \%$ C.L. $)$ & $\alpha(\mathrm{eV})$ \\
\hline MINOS [77] & $\tau_{3} / m_{3}>2.1 \times 10^{-12}$ & $\alpha_{3}<3.13 \times 10^{-4}$ \\
MINOS CC [37] & $\tau_{3} / m_{3}>2.0 \times 10^{-12}$ & $\alpha_{3}<3.29 \times 10^{-4}$ \\
MINOS CC + NC [37] & $\tau_{3} / m_{3}>2.8 \times 10^{-12}$ & $\alpha_{3}<2.35 \times 10^{-4}$ \\
T2K CC [37] & $\tau_{3} / m_{3} \sim 7.8 \times 10^{-13}-8.3 \times 10^{-12}$ & $\alpha_{3} \sim 7.9 \times 10^{-5}-8.4 \times 10^{-4}$ \\
MINOS + T2K [37] & $\tau_{3} / m_{3}>2.8 \times 10^{-12}$ & $\alpha_{3}<2.35 \times 10^{-4}$ \\
DUNE (invisible) [44] & $\tau_{3} / m_{3}>4.50 \times 10^{-11}$ & $\alpha_{3}<1.46 \times 10^{-5}$ \\
DUNE (visible) [43] & $\tau_{3} / m_{3}>1.95-2.6 \times 10^{-10}$ & $\alpha_{3}<2.53-3.37 \times 10^{-6}$ \\
Atmospheric + LBL [36] & $\tau_{3} / m_{3} \geq 2.9 \times 10^{-10}$ & $\alpha_{3}<2.27 \times 10^{-6}$ \\
ICAL (CC only) (our results) & $\tau_{3} / m_{3} \geq 1.51 \times 10^{-10}$ & $\alpha_{3}<4.36 \times 10^{-6}$ \\
Solar [22,24-26] & $\tau / m>9 \times 10^{-5}$ & $\alpha_{2}<7.3 \times 10^{-12}$ \\
ICE CUBE [39-42] & $\tau / m>10$ & $\alpha<6.58 \times 10^{-17}$ \\
AGN (100 Mpc, 1 TeV) [78] & $\tau / m>10^{4}$ & $\alpha<6.58 \times 10^{-20}$ \\
SN1987A (10 kpc, 10 MeV) [22,27,42,78] & $\tau / m>10^{5}$ & $\alpha<6.58 \times 10^{-21}$ \\
\hline \hline
\end{tabular}



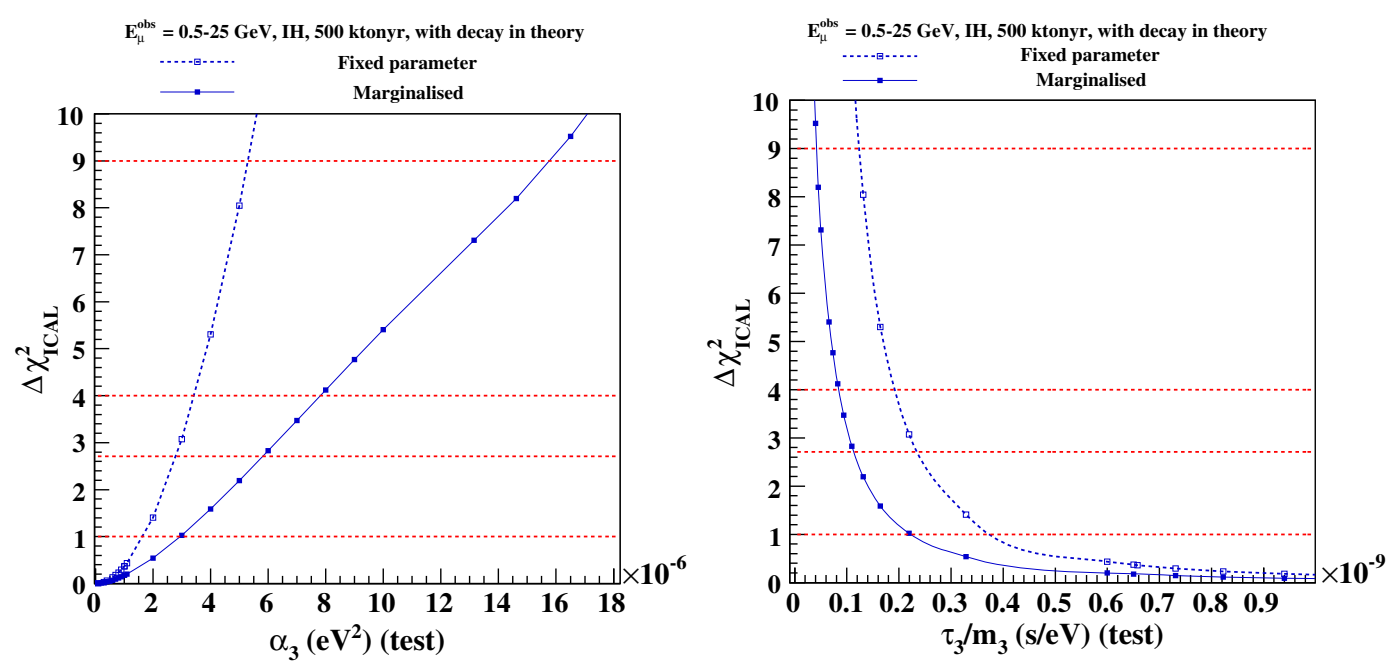

FIG. 5. Bounds on the allowed values of (left) $\alpha_{3} \mathrm{eV}^{2}$ (right) $\tau_{3} / m_{3}(\mathrm{~s} / \mathrm{eV})$ with 500 kton year exposure of ICAL with IH as true hierarchy. The comparison of results for fixed parameter and marginalized cases is shown.

dashed curves gives us an idea of the impact of marginalization over the oscillation parameters on the sensitivity of the experiment to decay. From Fig. 4 it can be seen that with marginalization of the oscillation parameters, the sensitivity decreases as expected. The right panel shows the sensitivity to decay in terms of $\tau_{3} / m_{3}$ in $\mathrm{s} / \mathrm{eV}$. The expected sensitivity of ICAL to $\alpha_{3}$ is shown in Table III. The corresponding values of $\tau_{3} / m_{3}$ in units of s/eV are also given. Note that by sensitivity limit we mean the value of $\alpha_{3}$ $\left(\tau_{3} / m_{3}\right)$ up to which ICAL can rule out neutrino decay.

The lower bound on $\tau_{3} / m_{3}$ for the invisible decay scenario from MINOS data was shown to be $\tau_{3} / m_{3}>$ $2.8 \times 10^{-12}(\mathrm{~s} / \mathrm{eV})$ at $90 \%$ C.L. This corresponds to an upper limit $\alpha_{3}<2.35 \times 10^{-4} \mathrm{eV}^{2}$. Table III shows that ICAL is expected to tighten these bounds by 2 orders of magnitude with just charged current $\nu_{\mu}$ and $\bar{\nu}_{\mu}$ events. At 90\% C.L., ICAL with marginalization is expected to give a lower bound of $\tau_{3} / m_{3}>1.51 \times 10^{-10}(\mathrm{~s} / \mathrm{eV})$ which corresponds to $\alpha_{3}<4.36 \times 10^{-6} \mathrm{eV}^{2}$. A comparison of the bounds on neutrino decay from various neutrino sources and experiments are shown in Table IV.

The expected sensitivity with fixed parameters as well as marginalization for true $\mathrm{IH}$ are shown in Fig. 5. At $90 \%$ C.L., the upper bound on $\alpha_{3}$ is $\alpha_{3}<2.78 \times 10^{-6} \mathrm{eV}^{2}$ with fixed parameters and $\alpha_{3}<5.82 \times 10^{-6} \mathrm{eV}^{2}$ with marginalization. These are only slightly worse than the sensitivities obtained with true NH. In terms of $\tau_{3} / m_{3}$, these limits translate as the lower limits $\tau_{3} / m_{3}>2.42 \times 10^{-10}$ and $\tau_{3} / m_{3}>1.14 \times 10^{-10} \mathrm{~s} / \mathrm{eV}$ for the fixed parameter and marginalized cases, respectively. The expected sensitivity to $\alpha_{3}$ at different C.L. with true $\mathrm{IH}$ is summarized in Table V.

The analysis discussed above gives us the sensitivity to $\alpha_{3}$ when we fit a data with no decay with a theory which has decay. On the other hand, if neutrinos indeed decay into sterile components, and if the decay rate is large enough to be observed in ICAL, we will be able to discover neutrino decay at this experiment. Therefore, we next estimate how much the decay rate needs to be in order for ICAL to make this discovery. For this analysis, we simulate the data with different values of $\alpha_{3}$ and fit it with a theory with no decay. The analysis was done for $500 \mathrm{kton}-\mathrm{yr}$ exposure of ICAL for fixed parameters as well as with marginalization of the undisplayed parameters over their respective $3 \sigma$ ranges. The results are shown in Fig. 6 by the red-dashed curve for the fixed parameter case and the red-solid line for the marginalized case. However, we find that for the discovery potential, the marginalization has no effect and gives the same result as the fixed parameter case. We find that ICAL will be able to discover neutrino decay at the $90 \%$ C.L. if $\alpha_{3}>2.5 \times 10^{-6} \mathrm{eV}^{2}$. We also plot the sensitivity curves, blue dashed (solid) lines for the fixed parameter (marginalized) case, in this figure for a comparison between the "sensitivity" and "discovery" potential of $\alpha_{3}$. We can see that the sensitivity and discovery limits of ICAL are very

TABLE V. Sensitivity to $\alpha_{3}\left(\mathrm{eV}^{2}\right)$ and $\tau_{3} / m_{3}(\mathrm{~s} / \mathrm{eV})$ with 500 kton year exposure of ICAL assuming IH as the true hierarchy.

\begin{tabular}{llcl}
\hline \hline Analysis type & $\chi^{2}$ & $\alpha_{3}\left(\mathrm{eV}^{2}\right)$ & $\tau_{3} / m_{3}(\mathrm{~s} / \mathrm{eV})$ \\
\hline Fixed parameters & 1 & $1.65 \times 10^{-6}$ & $4.35 \times 10^{-10}$ \\
& 2.71 & $2.78 \times 10^{-6}$ & $2.42 \times 10^{-10}$ \\
& 4 & $3.43 \times 10^{-6}$ & $1.97 \times 10^{-10}$ \\
& 9 & $5.31 \times 10^{-6}$ & $1.25 \times 10^{-10}$ \\
Marginalized & 1 & $2.97 \times 10^{-6}$ & $2.21 \times 10^{-10}$ \\
& 2.71 & $5.82 \times 10^{-6}$ & $1.14 \times 10^{-10}$ \\
& 4 & $7.82 \times 10^{-6}$ & $8.44 \times 10^{-11}$ \\
& 9 & $1.58 \times 10^{-5}$ & $4.21 \times 10^{-11}$ \\
\hline \hline
\end{tabular}




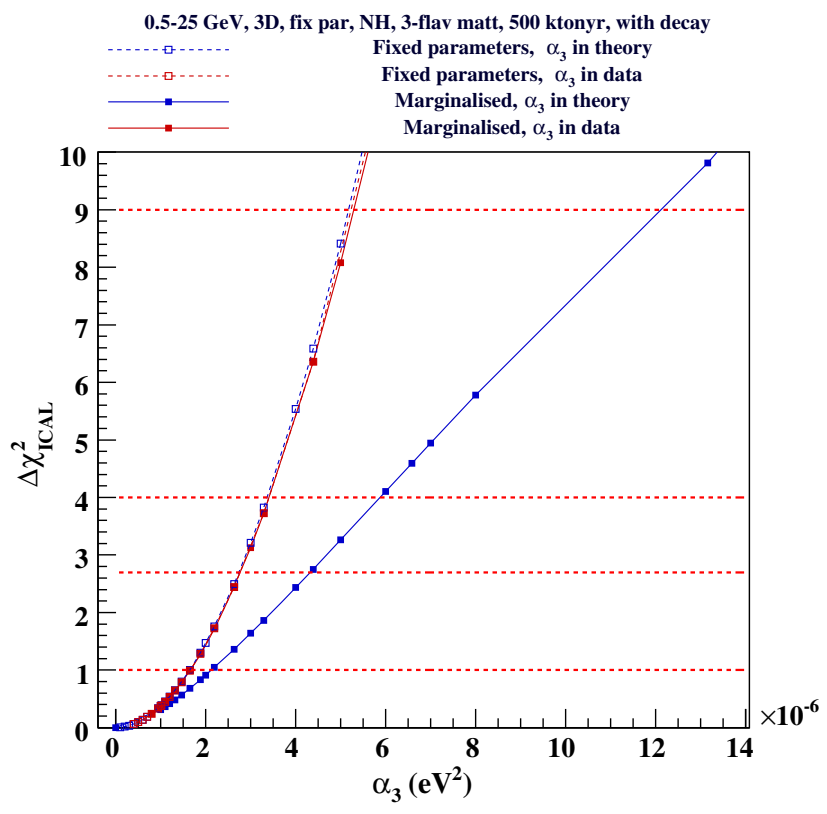

FIG. 6. Discovery potential of $\alpha_{3}$ by ICAL with 500 kton year exposure assuming $\mathrm{NH}$ as the true hierarchy, from fixed parameter and marginalized analyses.

similar for fixed parameter analysis. However, for the marginalized case the "discovery potential" is significantly higher than the sensitivity limit and is the same as the fixed parameter case.

The reason why the expected sensitivity limit worsens due to marginalization while the expected discovery limit does not can be understood as follows. For the sensitivity analysis we generate the data for no decay and $\theta_{23}$ maximal and fit it with a theory where $\alpha_{3} \neq 0$. Since the effect of decay is to reduce the number of events and suppress the event spectrum for fixed parameter there will be a difference between the data and the theory giving a higher $\chi^{2}$. For the marginalized case, this can be compensated to some extent by suitably changing the value of $\theta_{23}$ from maximal and thereby reducing $\sin ^{2} 2 \theta_{23}$, the leading term that controls the amplitude of oscillations in the case of muon neutrino survival probability. This can be seen in Fig. 7. In this figure the solid line denotes the data generated with $\alpha_{3}=0$, i.e no decay and $\theta_{23}=45^{\circ}$ while the dashed (dotted) lines show the theory events for a nonzero $\alpha_{3}$ and $\theta_{23}=45^{\circ}\left(38.65^{\circ}\right)$. We can see that the lower value of $\theta_{23}$ compensates for the depletion due to decay and can give a lower $\chi^{2}$. As a result the expected sensitivity drops when the sensitivity $\chi^{2}$ is marginalized over $\theta_{23}$.

On the other hand, for the expected discovery limit case we generate the data for nonzero $\alpha_{3}$ and maximal mixing and fit it with a theory with no decay. In this case, the data has events lower than the theory due to decay. This can be seen from the second panel of Fig. 7 where the blue (red) solid line denotes the data events for muon neutrinos (antineutrinos). However, unlike the sensitivity case, here one cannot change $\theta_{23}$ to reduce the event spectrum any further to compensate for the difference between data and theory since maximal mixing already corresponds to maximal suppression of the muon neutrino survival probability, the leading oscillation channel for atmospheric neutrinos. As a result, the fit continues to keep $\theta_{23}$ at its maximal value and marginalization fails to lower the $\chi^{2}$ any
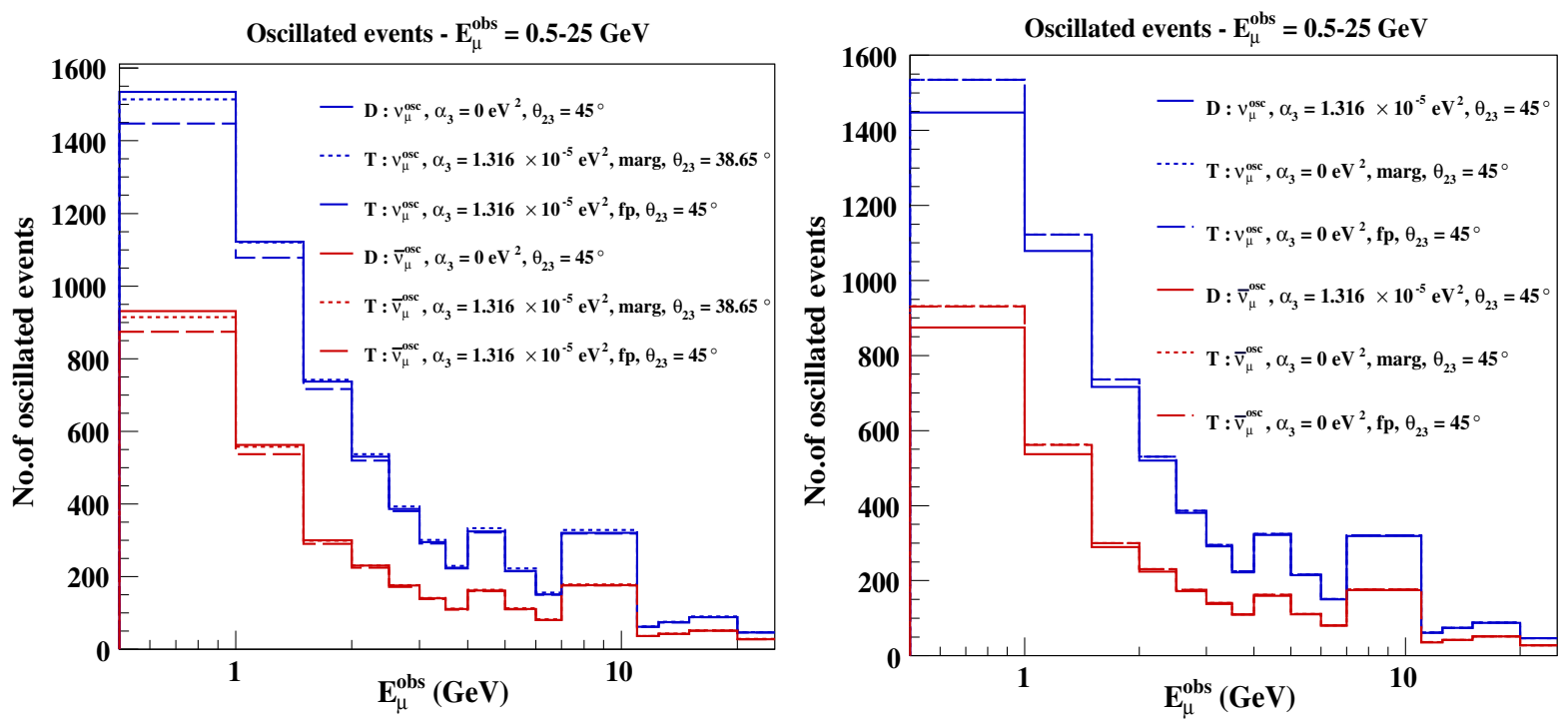

FIG. 7. Number of oscillated events per $E_{\mu}^{\mathrm{obs}}$ bin from $0.5-25 \mathrm{GeV}$ (left) with $\alpha_{3}=0 \mathrm{eV}^{2}$ in data and $\alpha_{3}=1.316 \times 10^{-5} \mathrm{eV}^{2}$ in theory; (right) with $\alpha_{3}=1.316 \times 10^{-5} \mathrm{eV}^{2}$ in data and $\alpha_{3}=0 \mathrm{eV}^{2}$ in theory for the marginalized case. "D" represents data and " $\mathrm{T}$ " represents theory events. The blue histograms are for $\nu_{\mu}$ and the red ones are for $\bar{\nu}_{\mu}$ events. The theory events are generated with marginalization of parameters except $\alpha_{3}$ in their respective $3 \sigma$ ranges. 

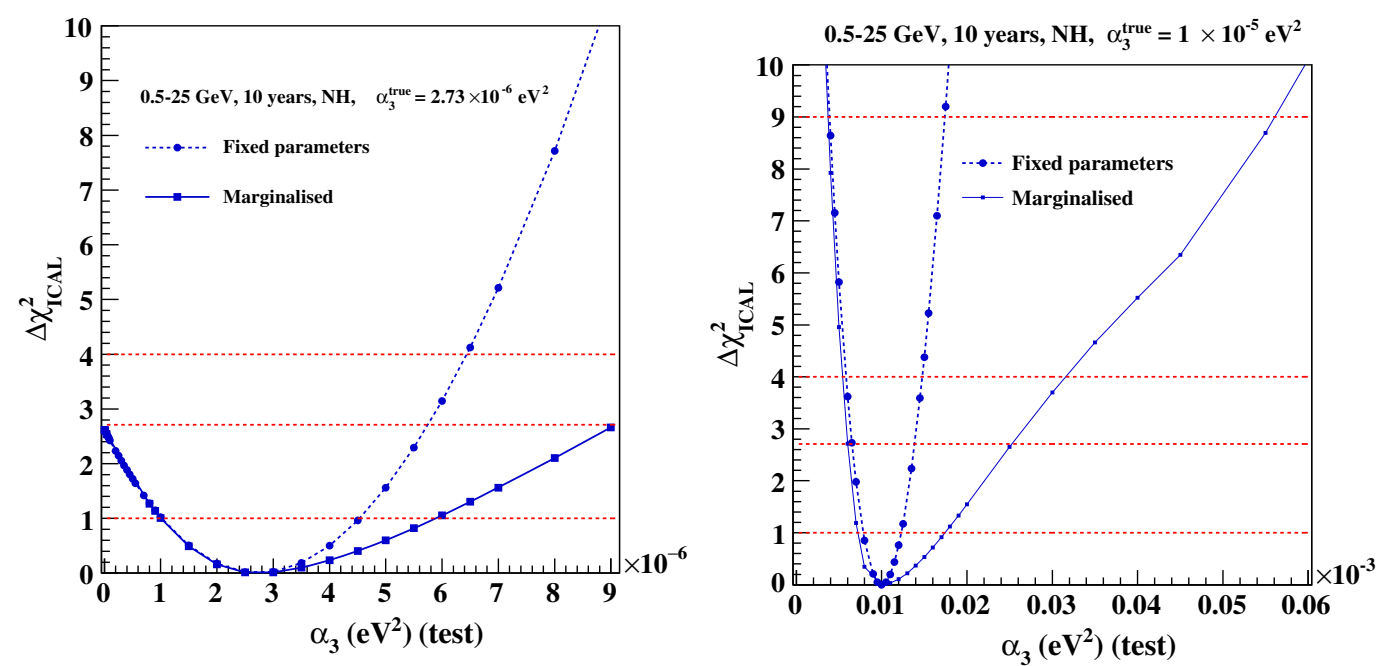

FIG. 8. Precision on $\alpha_{3}$ with (left) true $\alpha_{3}=2.73 \times 10^{-6} \mathrm{eV}^{2}$ and (right) true $\alpha_{3}=1.0 \times 10^{-5} \mathrm{eV}^{2}$. A comparison of the fixed parameter as well as marginalized analyses are shown. NH is taken as the true hierarchy.

further. This can also be seen from Fig. 7 where the dotted line shows the theory events obtained after marginalization and this is higher than the data events and the same as the fixed parameter case.

\section{Precision measurement of $\alpha_{3}$}

Next we present the results on the precision measurement of $\alpha_{3}$ with 500 kton year exposure of ICAL with true NH. Two true values of $\alpha_{3}$ are considered; i.e., $\alpha_{3}=$ $2.73 \times 10^{-6}$ and $1 \times 10^{-5} \mathrm{eV}^{2}$. The first true value corresponds to the $90 \%$ C.L. obtained from the fixed parameter sensitivity analysis as described in Sec. IV B. The second value is chosen to be a much higher value than the first one. The results of the analyses are shown in Fig. 8.

The minimum and maximum values of $\alpha_{3}$ at $1 \sigma$ for fixed parameter and marginalized cases for true $\alpha_{3}=$ $2.73 \times 10^{-6}$ and $1 \times 10^{-5} \mathrm{eV}^{2}$ are given in Table VI.

How well can ICAL exclude the no-decay scenario depends on the true value of $\alpha_{3}$. From Fig. 8, it can be seen that if the true value of $\alpha_{3}$ is very small, the no-decay scenario cannot be excluded well. For $\alpha_{3}^{\text {true }}=2.73 \times 10^{-6} \mathrm{eV}^{2}$, the no-decay case can only be excluded till $90 \%$ CL for both fixed and marginalised analyses. If the true value of $\alpha_{3}$ is

TABLE VI. Minimum and maximum values of $\alpha_{3}$ at $1 \sigma$ for $\alpha_{3}^{\text {true }}=2.73 \times 10^{-6}$ and $1 \times 10^{-5} \mathrm{eV}^{2}$ for fixed parameter and marginalized analyses with true $\mathrm{NH}$.

\begin{tabular}{lccc}
\hline \hline Analysis type & $\begin{array}{c}\alpha_{3_{\text {true }}} \\
\times 10^{-6} \mathrm{eV}^{2}\end{array}$ & $\begin{array}{c}\alpha_{3_{\text {min }}}(1 \sigma) \\
\times 10^{-6} \mathrm{eV}^{2}\end{array}$ & $\begin{array}{c}\alpha_{3_{\text {max }}}(1 \sigma) \\
\times 10^{-6} \mathrm{eV}^{2}\end{array}$ \\
\hline FP & 2.73 & 1.02 & 4.53 \\
Marginalized & & 1.01 & 5.89 \\
FP & 10.0 & 7.85 & 12.34 \\
Marginalized & & 7.19 & 17.46 \\
\hline \hline
\end{tabular}

$1.0 \times 10^{-5} \mathrm{eV}^{2}$, then no-decay can be excluded at $3 \sigma$. The lesser the value of $\alpha_{3}^{\text {true }}$, the more difficult it becomes to exclude the no-decay case with ICAL with 500 kton year exposure.

\section{PRECISION MEASUREMENT OF $\sin ^{2} \theta_{23}$ AND $\left|\Delta m_{32}^{2}\right|$}

We next look at the impact of neutrino decay on the precision measurement of the mixing angle $\theta_{23}$ and the mass squared difference $\left|\Delta m_{32}^{2}\right|$ at ICAL. A comparison of the precision measurement in the presence and absence of decay is presented. In the no decay case both data and theory are generated without the decay parameter and in the case with decay both data and theory are generated with nonzero values of $\alpha_{3}$. For all results presented in this section, the value $\alpha_{3}=1 \times 10^{-5} \mathrm{eV}^{2}$ is used to generate the data. In the fixed parameter analysis this is kept fixed in theory and for the marginalized case, the range over which $\alpha_{3}$ is marginalized is taken to be $\alpha_{3}=\left[0,2.35 \times 10^{-4}\right] \mathrm{eV}^{2}$ which corresponds to the $90 \%$ C.L. bound given by the MINOS analysis. The other parameters are kept fixed at their true values as shown in Table II for the fixed parameter analyses and varied in the $3 \sigma$ ranges as shown in the same table for the marginalized case. The $1 \sigma$ precision on a parameter $\lambda$ is defined as

$$
p(\lambda)=\frac{\lambda_{\max -2 \sigma}-\lambda_{\min -2 \sigma}}{4 \lambda_{\text {true }}},
$$

where $\lambda_{\max -2 \sigma}$ and $\lambda_{\min -2 \sigma}$ are the maximum and minimum allowed values of $\lambda$ at $2 \sigma$ and $\lambda_{\text {true }}$ is the true choice.

\section{A. Precision on $\sin ^{2} \theta_{23}$ in the presence of decay}

The sensitivity to $\sin ^{2} \theta_{23}$ in the presence and absence of $\nu_{3}$ decay is shown in Fig. 9. The left panel shows the fixed 

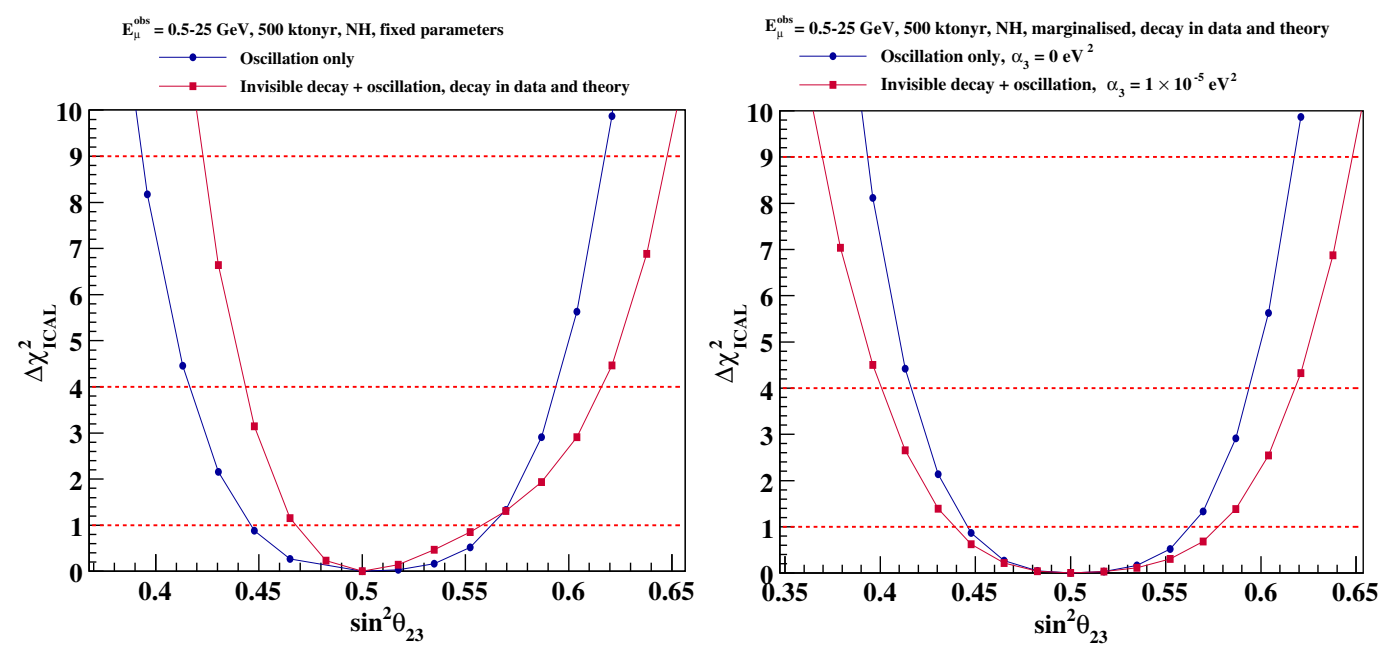

FIG. 9. Precision on $\sin ^{2} \theta_{23}$ in the presence and absence of invisible decay for (left) fixed parameter case (right) marginalized case. The value of decay parameter $\alpha_{3}$ in data is taken to be $1 \times 10^{-5} \mathrm{eV}^{2}$.

parameter results whereas the right panel shows the results for the marginalized case. For the fixed parameter case, in the absence of decay, the $1 \sigma$ precision on $\sin ^{2} \theta_{23}$ is $\sim 8.9 \%$. In presence of decay the $1 \sigma$ precision is $\sim 8.6 \%$ which is similar to the no decay case. However, it is important to note that even though the percentage precision is the same, the allowed parameter space is shifted to the right when there is decay, as compared to the no decay case. The minimum and maximum values of $\sin ^{2} \theta_{23}$ at $2 \sigma \mathrm{s}$ in the presence and absence of decay are shown in Table VII.

In order to understand the shift of parameter space, we show in Fig. 10 the number of oscillated $\nu_{\mu}$ and $\bar{\nu}_{\mu}$ events for three different values of $\theta_{23}-9^{\circ}, 45^{\circ}$ and $52^{\circ}$ and two different $\alpha_{3}-0$ and $1 \times 10^{-5} \mathrm{eV}^{2}$. Here $39^{\circ}$ and $52^{\circ}$ are representative values for lower octant and higher octant, respectively. We plot the events as a function of energy integrating over the zenith-angle bins. From the figures it can be seen that both in the absence and presence of decay there are differences between the number of events for various $\theta_{23}$ values. For the case of no decay this difference is less as compared to the case where decay is present. Comparing the figures on the left and right panels one also observes that the difference between the number of events

TABLE VII. Minimum and maximum values of $\sin ^{2} \theta_{23}$ at $2 \sigma$, with and without decay for fixed parameter and marginalized cases. The relative $1 \sigma$ precision obtained is also shown. $\mathrm{NH}$ is taken as the true hierarchy.

\begin{tabular}{lccc}
\hline \hline Analysis type & $\sin ^{2} \theta_{23_{\min }}(2 \sigma)$ & $\sin ^{2} \theta_{23_{\max }}(2 \sigma)$ & $\begin{array}{c}\text { Precision } \\
\text { at } 1 \sigma(\%)\end{array}$ \\
\hline$\alpha_{3}=0 \mathrm{eV}^{2}(\mathrm{fp})$ & 0.416 & 0.594 & 8.9 \\
$\alpha_{3}=1 \times 10^{-5} \mathrm{eV}^{2}(\mathrm{fp})$ & 0.444 & 0.616 & 8.6 \\
$\alpha_{3}=0 \mathrm{eV}^{2}(\mathrm{marg})$ & 0.416 & 0.594 & 8.9 \\
$\alpha_{3}=1 \times 10^{-5} \mathrm{eV}^{2}$ (marg) & 0.401 & 0.618 & 10.85 \\
\hline \hline
\end{tabular}

for $\theta_{23}=39^{\circ}$ and $45^{\circ}$ is more in the presence of decay and the curve for $\theta_{23}=45^{\circ}$ is closer to $52^{\circ}$. Now, in obtaining the precision plot the data is generated with true $\theta_{23}=45^{\circ}$ and in theory the $\theta_{23}$ is kept fixed. For $\theta_{23}$ in the lower octant the difference of the number of events with that for $45^{\circ}$ being more in the presence of decay, the $\chi^{2}$ for a $\theta_{23}$ in the lower octant will be higher as compared to the no decay case. On the other hand, for $\theta_{23}$ in the higher octant the difference in the number of events with $\theta_{23}=45^{\circ}$ being less in the presence of decay, one gets a lower $\chi^{2}$ as compared to the no decay case. This explains why the precision curve shifts towards higher $\theta_{23}$ values.

For the marginalized case, in the presence of decay the overall precision becomes worse compared to the no decay case. The $1 \sigma$ precision when decay is present is $\sim 10.85 \%$ whereas for no decay it is $\sim 8.9 \%$. This can be explained as follows. In the marginalized case, for only oscillation we are trying to fit the data generated with $\theta_{23}=45^{\circ}$, varying the other parameters in theory. In this case the $\theta_{13}$ can be adjusted to give a slightly lower $\chi^{2}$. In the presence of decay we generate the data for a particular nonzero $\alpha_{3}$ and $\theta_{23}=45^{\circ}$. But now in theory we vary $\alpha_{3}$ as well as the other parameters. For $\theta_{23}$ in the lower octant, the theory events will be higher than the data events as can be seen by comparing the events in the second panel of Fig. 10. However, in this case the $\alpha_{3}$ can be increased to give a better fit and a lower $\chi^{2}$. On the other hand for $\theta_{23}$ in the higher octant, the data events are higher than the theory events and $\alpha_{3}$ can be decreased in theory to match the data better and give a lower $\chi^{2}$. This explains the widening of the $\chi^{2}$ vs $\theta_{23}$ curve in the presence of decay. Note that this is more for the higher octant because the difference of the events for $\theta_{23}=45^{\circ}$ and say $52^{\circ}$ is less as compared to $\theta_{23}$ in the lower octant, say $39^{\circ}$. This gives a lower $\chi^{2}$ thus allowing more $\theta_{23}$ values in the higher octant. 

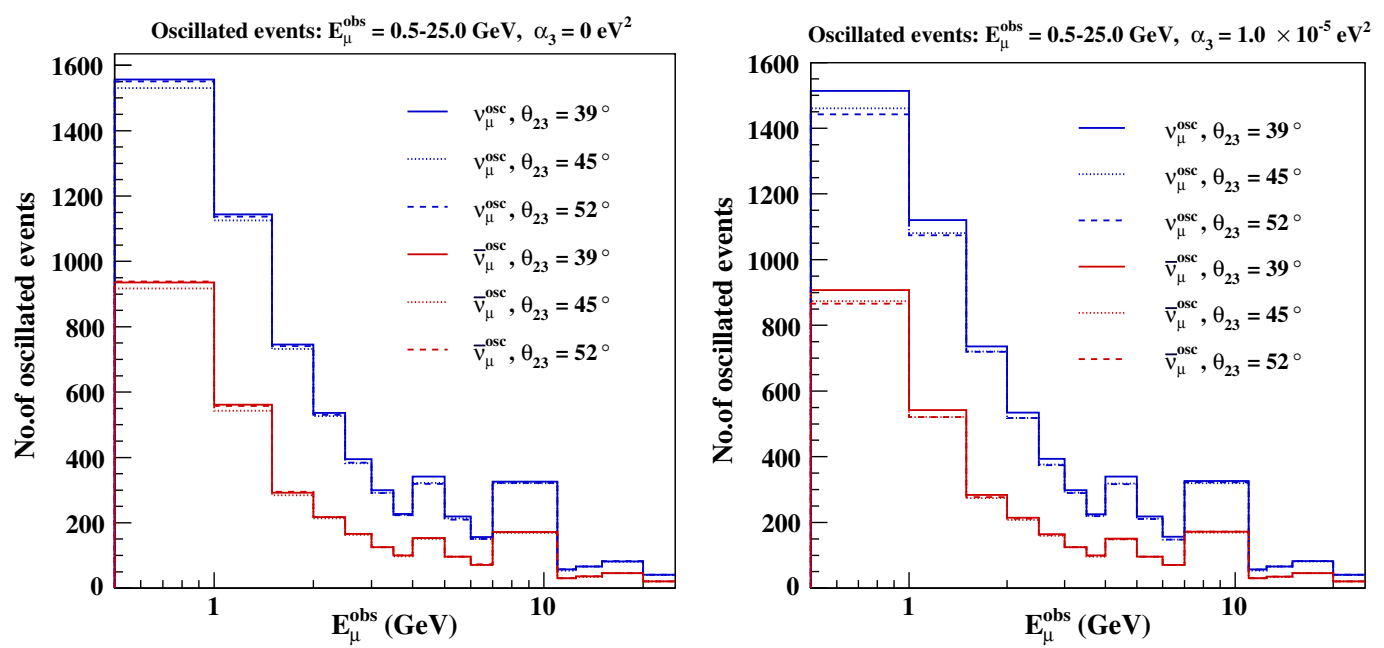

FIG. 10. Oscillated $\nu_{\mu}$ events as a function of $E_{\mu}$ for (left) $\alpha_{3}=0 \mathrm{eV}^{2}$ and (right) $\alpha_{3}=1 \times 10^{-5} \mathrm{eV}^{2}$ for $\theta_{23}=39,45$ and $52^{\circ}$.
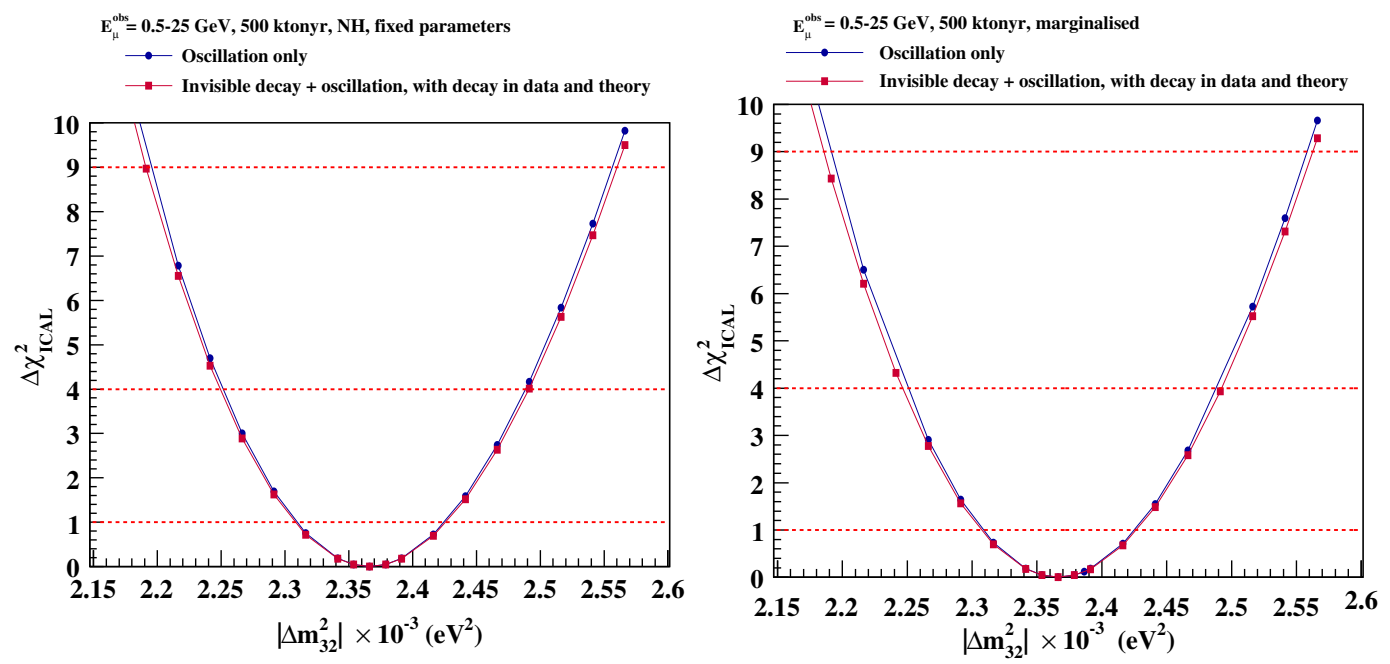

FIG. 11. Precision on $\left|\Delta m_{32}^{2}\right|$ in the absence and presence of invisible decay (left) fixed parameters (right) with marginalization.

\section{B. Precision on $\left|\Delta m_{32}^{2}\right|$ in the presence of decay}

The precision on the magnitude of the mass square difference $\left|\Delta m_{32}^{2}\right|$ in the presence and absence of invisible decay of $\nu_{3}$ is presented in Fig. 11. NH is taken as the true hierarchy. The relative $1 \sigma$ precision on $\left|\Delta m_{32}^{2}\right|$ with oscillations only and with decay is $\sim 2.5 \%$ for the fixed parameter case. When marginalization is done this becomes $\sim 2.6 \%$ for both cases. Thus it can be seen that the presence of decay does not affect the precision on $\left|\Delta m_{32}^{2}\right|$ much. This is because decay mainly affects the amplitude of the oscillations and not the phase which is determined by $\left|\Delta m_{32}^{2}\right|$. The minimum and maximum values of $\sin ^{2} \theta_{23}$ at $2 \sigma \mathrm{s}$ in the presence and absence of decay are shown in Table VIII.
TABLE VIII. Minimum and maximum values of $\left|\Delta m_{32}^{2}\right|$ at $2 \sigma$, with and without decay for fixed parameter and marginalized cases. The relative $1 \sigma$ precision obtained is also shown. $\mathrm{NH}$ is taken as the true hierarchy.

\begin{tabular}{lccc}
\hline \hline Analysis type & $\begin{array}{c}\left|\Delta m_{32}^{2}\right|_{\min }(2 \sigma) \\
\times 10^{-3} \mathrm{eV}^{2}\end{array}$ & $\begin{array}{c}\left|\Delta m_{32}^{2}\right|_{\max }(2 \sigma) \\
\times 10^{-3} \mathrm{eV}^{2}\end{array}$ & $\begin{array}{c}\text { Precision } \\
\text { at } 1 \sigma(\%)\end{array}$ \\
\hline$\alpha_{3}=0 \mathrm{eV}^{2}(\mathrm{fp})$ & 2.252 & 2.489 & 2.5 \\
$\alpha_{3}=1 \times 10^{-5} \mathrm{eV}^{2}$ & 2.249 & 2.492 & 2.5 \\
$\quad(\mathrm{fp})$ & & & \\
$\alpha_{3}=0 \mathrm{eV}^{2}(\mathrm{marg})$ & 2.252 & 2.489 & 2.6 \\
$\alpha_{3}=1 \times 10^{-5} \mathrm{eV}^{2}$ & 2.247 & 2.493 & 2.6 \\
$\quad$ (marg) & & & \\
\hline \hline
\end{tabular}




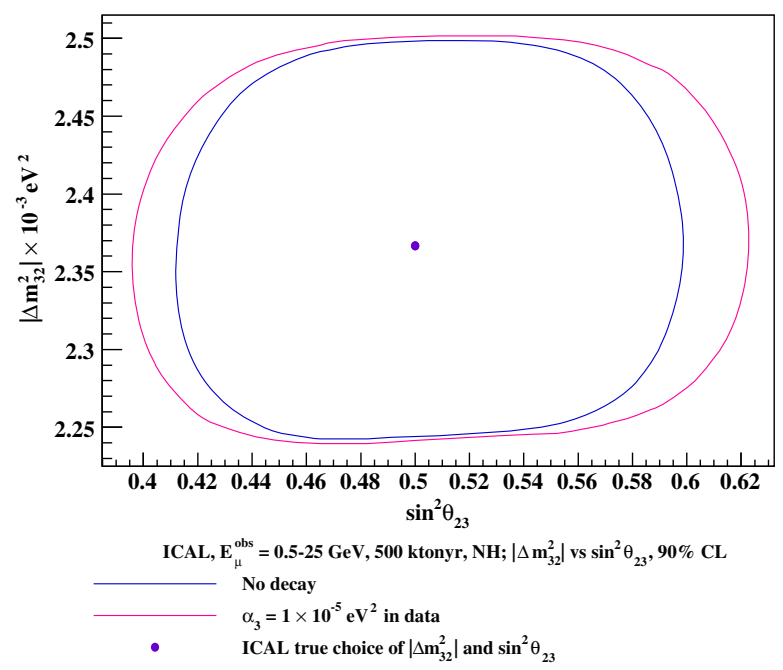

FIG. 12. Expected $90 \%$ C.L. contour in the $\sin ^{2} \theta_{23}-\left|\Delta m^{2}{ }_{32}\right|$ plane, with and without decay, for NH. The value of $\alpha_{3}$ in data is taken as $1 \times 10^{-5} \mathrm{eV}^{2}$.

\section{Simultaneous precision on $\sin ^{2} \theta_{23}$ and $\left|\Delta m_{32}^{2}\right|$ in the presence of $\alpha_{3}$}

In this section the expected C.L. contours in the $\sin ^{2} \theta_{23}-\left|\Delta m_{32}^{2}\right|$ plane in the presence of decay are shown. The results are shown for true $\mathrm{NH}$. A value of decay parameter $\alpha_{3}=1 \times 10^{-5} \mathrm{eV}^{2}$ is taken in data and is marginalized in the $3 \sigma$ range $\left[0,2.35 \times 10^{-4}\right] \mathrm{eV}^{2}$. The other parameters are also marginalized over their $3 \sigma$ ranges as before. The expected $90 \%$ C.L. contour in the $\sin ^{2} \theta_{23}-$ $\left|\Delta m_{32}^{2}\right|$ plane in the presence and absence of decay is shown in Fig. 12.

It can be seen that the precision worsens in the presence of decay. The contour widens significantly along the $\sin ^{2} \theta_{23}$ axis, more so in the second octant for the same reason as explained in the context of marginalized case in Fig. 9. In the absence of decay the precision on $\sin ^{2} \theta_{23}$ at $90 \%$ C.L. is $18.5 \%$. This worsens to $22.3 \%$ with a decay parameter $\alpha_{3}=1 \times 10^{-5} \mathrm{eV}^{2}$. The precision on $\left|\Delta m_{32}^{2}\right|$ worsens only marginally from the no decay value of $5.35 \%$ to $5.46 \%$ for the same central value of $\alpha_{3}$. This is expected since the decay affects the oscillation amplitude which in turn affects the precision on $\sin ^{2} \theta_{23}$.

\section{SUMMARY AND DISCUSSIONS}

The expected sensitivity of ICAL to the decay lifetime of the mass eigenstate $\nu_{3}$, when it decays via the invisible decay mode was presented. The analysis was performed in the three-generation neutrino oscillation framework including decay as well as Earth matter effects. The decay was parametrized in terms of $\alpha_{3}=m_{3} / \tau_{3}$, where $m_{3}$ is the mass and $\tau_{3}$ the lifetime at rest of the mass eigenstate $\nu_{3}$. With
500 kton - yr of exposure, ICAL is expected to constrain the invisible decay rate to $\alpha_{3}<4.36 \times 10^{-6} \mathrm{eV}^{2}$ at $90 \%$ C.L., which is 2 orders of magnitude tighter than the bound obtained in [37] for MINOS. In [37] both charged current (CC) and neutral current (NC) events were considered whereas in our study only atmospheric $\mathrm{CC} \nu_{\mu}$ and $\bar{\nu}_{\mu}$ events were used. For invisible neutrino decay, the NC background will be less. Hence the sensitivity to $\alpha_{3}$ is expected to improve.

The effect of decay on the 2-3 oscillation parameters was also studied. Since the amplitude of oscillations is affected most by the presence of decay, it was found that decay affected the precision measurement of $\sin ^{2} \theta_{23}$. For 500 kton-yrs of exposure assuming $\mathrm{NH}$ as the true hierarchy, the $1 \sigma$ precision on $\sin ^{2} \theta_{23}$ was found to worsen to $10.85 \%$ when $\alpha_{3}=10^{-5} \mathrm{eV}^{2}$ was assumed. This is worse as compared to the $8.87 \%$ obtained with the oscillation only hypothesis. In the case of $\left|\Delta m_{32}^{2}\right|$ the $1 \sigma$ precision without decay is $2.5 \%$ whereas the inclusion of invisible decay does not affect it at all. The effect of $\alpha_{3}$ on the sensitivity to neutrino mass hierarchy and octant of $\theta_{23}$ will be studied elsewhere [79].

It is also noteworthy that the sensitivity to smaller $\alpha_{3}$ comes mainly from the lower energy bins below $2 \mathrm{GeV}$. Hence, if we can improve the efficiencies and resolutions of the detector, especially for muons in the lower energy region, we will be able to put a better limit on $\alpha_{3}$. Reduction of the energy threshold for the detection of low energy neutrinos in the future will also help probing phenomena like decay with increased precision. This is important since the atmospheric neutrino flux peaks at lower energies and by being able to detect and analyze more events we will further improve our sensitivities to all parameters including $\alpha_{3}$.

\section{ACKNOWLEDGMENTS}

C. Gupta thanks the India-based Neutrino Observatory collaboration, which is jointly funded by the Department of Atomic Energy, India and Department of Science and Technology, India, for the financial support. The authors acknowledge Prof. Amol Dighe, Tata Institute of Fundamental Research, Mumbai and Prof. D. Indumathi, Institute of Mathematical Sciences (IMSc), Chennai for the discussions. We also acknowledge our INO internal referees, Prof. S. Uma Sankar, Indian Institute of Technology Bombay, Mumbai and Prof. Jim Libby, Indian Institute of Technology Madras, Chennai for their comments and suggestions. S. M. Lakshmi acknowledges Nandadevi and Satpura clusters belonging to the computing facility of IMSc Chennai, with which this analysis was made possible. 
[1] I. Esteban, M. C. Gonzalez-Garcia, M. Maltoni, I. MartinezSoler, and T. Schwetz, Updated fit to three neutrino mixing: exploring the accelerator-reactor complementarity, J. High Energy Phys. 01 (2017) 087.

[2] K. Nakamura and S. T. Petcov (Neutrino mass, mixing, and oscillations, Review in Particle Data Group), Review of particle physics, Chin. Phys. C 40, 100001 (2016).

[3] R. Acciarri et al. (DUNE Collaboration), Long-Baseline Neutrino Facility (LBNF) and Deep Underground Neutrino Experiment (DUNE) Conceptual Design Report Volume 2: The Physics Program for DUNE at LBNF, arXiv: 1512.06148 .

[4] K. Abe et al. (Hyper-Kamiokande Proto- Collaboration), Physics potential of a long-baseline neutrino oscillation experiment using a J-PARC neutrino beam and hyperKamiokande, Prog. Theor. Exp. Phys. 2015, $053 \mathrm{C} 02$ (2015).

[5] Y. Li, J. Cao, Y. Wang, and L. Zhan, Unambiguous determination of the neutrino mass hierarchy using reactor neutrinos, Phys. Rev. D 88, 013008 (2013).

[6] S. B. Kim (RENO Collaboration), New results from RENO and prospects with RENO-50, Nucl. Part. Phys. Proc. 265, 93 (2015).

[7] M. G. Aartsen et al. (IceCube-Pingu Collaboration), Letter of Intent: The Precision IceCube Next Generation Upgrade (PINGU), arXiv:1401.2046.

[8] S Adrián-Martínez et al., Letter of intent for KM3NeT 2.0, J. Phys. G 43, 084001 (2016).

[9] A. Kumar, A. M. Vinod Kumar, A. Jash et al., Invited review: Physics potential of the ICAL detector at the India-based Neutrino Observatory (INO), Pramana J. Phys. 88, 79 (2017).

[10] M.S. Athar et al., India-based Neutrino Observatory: Project report, Volume I, INO-2006-01, 2006.

[11] A. Mirizzi, D. Montanino, and P. D. Serpico, Revisiting cosmological bounds on radiative neutrino lifetime, Phys. Rev. D 76, 053007 (2007).

[12] Y. Chikashige, R. N. Mohapatra, and R. D. Peccei, Are there real goldstone bosons associated with broken lepton number?, Phys. Lett. B 98, 265 (1981).

[13] G. B. Gelmini and M. Roncadelli, Left-handed neutrino mass scale and spontaneously broken lepton number, Phys. Lett. B 99, 411 (1981).

[14] G. B. Gelmini and J. W. F. Valle, Fast invisible neutrino decays, Phys. Lett. B 142, 181 (1984).

[15] A. Acker, S. Pakvasa, and James T. Pantaleone, Decaying Dirac neutrinos, Phys. Rev. D 45, R1 (1992).

[16] A. Acker, A. Joshipura, and S. Pakvasa, A neutrino decay model, solar antineutrinos and atmospheric neutrinos, Phys. Lett. B 285, 371 (1992).

[17] John N. Bahcall, N. Cabibbo, and A. Yahil, Are Neutrinos Stable Particles?, Phys. Rev. Lett. 28, 316 (1972).

[18] A. Acker and S. Pakvasa, Solar neutrino decay, Phys. Lett. B 320, 320 (1994).

[19] Z. G. Berezhiani, G. Fiorentini, M. Moretti, and A. Rossi, Fast neutrino decay and solar neutrino detectors, Z. Phys. C 54, 581 (1992).

[20] Z. G. Berezhiani, M. Moretti, and A. Rossi, Matter induced neutrino decay and solar antineutrinos, Z. Phys. C 58, 423 (1993).
[21] S. Choubey, S. Goswami, and D. Majumdar, Status of the neutrino decay solution to the solar neutrino problem, Phys. Lett. B 484, 73 (2000).

[22] A. Bandyopadhyay, S. Choubey, and S. Goswami, MSW mediated neutrino decay and the solar neutrino problem, Phys. Rev. D 63, 113019 (2001).

[23] A. S. Joshipura, E. Masso, and S. Mohanty, Constraints on decay plus oscillation solutions of the solar neutrino problem, Phys. Rev. D 66, 113008 (2002).

[24] A. Bandyopadhyay, S. Choubey, and S. Goswami, Neutrino decay confronts the SNO data, Phys. Lett. B 555, 33 (2003).

[25] J. M. Berryman, A. Gouvea, and D. Hernandez, Solar neutrinos and the decaying neutrino hypothesis, Phys. Rev. D 92, 073003 (2015).

[26] R. Picoreti, M. M. Guzzo, P. C. de Holanda, and O. L. G. Peres, Neutrino decay and solar neutrino seasonal effect, Phys. Lett. B 761, 70 (2016).

[27] J. A. Frieman, H. E. Haber, and K. Freese, Neutrino mixing, decays and supernova 1987A, Phys. Lett. B 200, 115 (1988).

[28] J. M. LoSecco, What the Atmospheric Neutrino Anomaly is Not, arXiv:hep-ph/9809499.

[29] P. Lipari and M. Lusignoli, Exotic solutions of the atmospheric neutrino problem, Phys. Rev. D 60, 013003 (1999).

[30] V. D. Barger, W. Y. Keung, and S. Pakvasa, Majoron emission by neutrinos, Phys. Rev. D 25, 907 (1982).

[31] V. D. Barger, J. G. Learned, S. Pakvasa, and T. J. Weiler, Neutrino Decay as an Explanation of Atmospheric Neutrino Observations, Phys. Rev. Lett. 82, 2640 (1999).

[32] G. L. Fogli, E. Lisi, A. Marrone, and G. Scioscia, SuperKamiokande data and atmospheric neutrino decay, Phys. Rev. D 59, 117303 (1999).

[33] S. Choubey and S. Goswami, Is neutrino decay really ruled out as a solution to the atmospheric neutrino problem from Super-Kamiokande data?, Astropart. Phys. 14, 67 (2000).

[34] V. D. Barger, J. G. Learned, P. Lipari, M. Lusignoli, S. Pakvasa, and T. J. Weiler, Neutrino decay and atmospheric neutrinos, Phys. Lett. B 462, 109 (1999).

[35] Y. Ashie et al., Evidence for an Oscillatory Signature in Atmospheric Neutrino Oscillation, Phys. Rev. Lett. 93, 101801 (2004).

[36] M. C. Gonzalez-Garcia and M. Maltoni, Status of oscillation plus decay of atmospheric and long-baseline neutrinos, Phys. Lett. B 663, 405 (2008).

[37] R. A. Gomes, A. L. G. Gomes, and O. L. G. Peres, Constraints on neutrino decay lifetime using long-baseline charged and neutral current data, Phys. Lett. B 740, 345 (2015).

[38] A. M. Gago, R. A. Gomes, A. L. G. Gomes, J. Jones-Perez, and O.L.G. Peres, Visible neutrino decay in the light of appearance and disappearance long-baseline experiments, J. High Energy Phys. 11 (2017) 022.

[39] J.F. Beacom, J.F. Beacom, N. F. Bell, D. Hooper, S. Pakvasa, and T. J. Weiler, Decay of High-Energy Astrophysical Neutrinos, Phys. Rev. Lett. 90, 181301 (2003).

[40] M. Maltoni and W. Winter, Testing neutrino flavor mixing plus decay with neutrino telescopes, J. High Energy Phys. 07 (2008) 064. 
[41] G. Pagliaroli, A. Palladino, F. L. Villante, and F. Vissani, Testing nonradiative neutrino decay scenarios with IceCube data, Phys. Rev. D 92, 113008 (2015).

[42] M. Bustamante, J. F. Beacom, and K. Murase, Testing decay of astrophysical neutrinos with incomplete information, Phys. Rev. D 95, 063013 (2017).

[43] P. Coloma and O. L. G. Peres, Visible neutrino decay at DUNE, arXiv:1705.03599.

[44] S. Choubey, S. Goswami, and D. Pramanik, A study of invisible neutrino decay at DUNE and its effects on $\theta_{23}$ measurement, J. High Energ. Phys. 02 (2018) 55.

[45] A. Chatterjee, K. K. Meghna, R. Kanishka, T. Thakore, V. Bhatnagar, R. Gandhi, D. Indumathi, N. K. Mondal, and N. Sinha, A simulations study of the muon response of the Iron Calorimeter detector at the India-based Neutrino Observatory, J. Instrum. 9, P07001 (2014).

[46] K. K. Meghna, PhD thesis, The Board of Studies in Physical Sciences, Homi Bhabha National Institute, 2015, http://www.hbni.ac.in/students/dsp_ths.html?nm=phys/ PHYS01200904012.pdf.

[47] R. Kanishka, K. K. Meghna, V. Bhatnagar, D. Indumathi, and N. Sinha, Simulations study of muon response in the peripheral regions of the Iron Calorimeter detector at the India-based Neutrino Observatory, J. Instrum. 10, P03011 (2015).

[48] K. Rawat, PhD thesis, Faculy of Science, Panjab University, Chandigarh, 2015, http://www.ino.tifr.res.in/ino/theses/ Kanishka_PhD_Thesis.pdf.

[49] M. M. Devi, A. Ghosh, D. Kaur, S. M. Lakshmi, S. Choubey, A. Dighe, D. Indumathi, S. Kumar, M. V. N. Murthy, and Md. Naimuddin, Hadron energy response of the Iron Calorimeter detector at the India-based Neutrino Observatory, J. Instrum. 8, P11003 (2013).

[50] S. M. Lakshmi, A Ghosh, M. M. Devi, D. Kaur, S. Choubey, A. Dighe, D. Indumathi, M. V. N. Murthy, and Md. Naimuddin, Simulation studies of hadron energy resolution as a function of iron plate thickness at INO-ICAL, J. Instrum. 9, T09003 (2014).

[51] L.S. Mohan, PhD thesis, The Board of Studies in Physical Sciences, Homi Bhabha National Institute, 2015, http://www.hbni.ac.in/students/dsp_ths.html?nm=phys/ PHYS01200904008.pdf.

[52] M. M. Devi, PhD thesis, The Board of Studies in Physical Sciences, Homi Bhabha National Institute, 2014, http://www.hbni.ac.in/students/dsp_ths.html?nm=phys/ PHYS01200904010.pdf.

[53] S. Agostinelli et al., Geant4-A simulation toolkit, Nucl. Instrum. Methods Phys. Res., Sect. A 506, 250 (2003).

[54] J. Allison et al., Geant4 developments and applications, IEEE Trans. Nucl. Sci. 53, 270 (2006).

[55] J. Allison et al., Recent developments in Geant4, Nucl. Instrum. Methods Phys. Res., Sect. A 835, 186 (2016).

[56] A. Ghosh, T. Thakore, and S. Choubey, Determining the neutrino mass hierarchy with INO, T2K, NO $\nu \mathrm{A}$ and reactor experiments, J. High Energy Phys. 04 (2013) 009.

[57] A. Ghosh and S. Choubey, Measuring the mass hierarchy with muon and hadron events in atmospheric neutrino experiments, J. High Energy Phys. 10 (2013) 174.
[58] M. M. Devi, T. Thakore, S. K. Agarwalla, and A. Dighe, Enhancing sensitivity to neutrino parameters at INO combining muon and hadron information, J. High Energy Phys. 10 (2014) 189.

[59] L. S. Mohan and D. Indumathi, Pinning down neutrino oscillation parameters in the 23 sector with a magnetized atmospheric neutrino detector: A new study, Eur. Phys. J. C 77, 54 (2017).

[60] A. Ajmi, A. Dev, M. Nizam, N. Nayak, and S. Uma Sankar, Improving the hierarchy sensitivity of ICAL using neural network, arXiv:1510.02350.

[61] T. Thakore, A. Ghosh, S. Choubey, and A. Dighe, The reach of INO for atmospheric neutrino oscillation parameters, J. High Energy Phys. 05 (2013) 058.

[62] D. Kaur, M. Naimuddin, and S. Kumar, The sensitivity of the ICAL detector at India-based Neutrino Observatory to neutrino oscillation parameters, Eur. Phys. J. C 75, 156 (2015).

[63] D. Kaur, Z. A. Dar, S. Kumar, and M. Naimuddin, Search for the differences in atmospheric neutrino and antineutrino oscillation parameters at the INO-ICAL experiment, Phys. Rev. D 95, 093005 (2017).

[64] M. Ghosh, P. Ghoshal, S. Goswami, N. Nath, and S. K. Raut, New look at the degeneracies in the neutrino oscillation parameters, and their resolution by $\mathrm{T} 2 \mathrm{~K}, \mathrm{NO} \nu \mathrm{A}$ and ICAL, Phys. Rev. D 93, 013013 (2016).

[65] A. Chatterjee, R. Gandhi, and J. Singh, Probing Lorentz and $C P T$ violation in a magnetized iron detector using atmospheric neutrinos, J. High Energy Phys. 06 (2014) 045 .

[66] N. Dash, V. M. Datar, and G. Majumder, Sensitivity for detection of decay of dark matter particle using ICAL at INO, Pramana 86, 927 (2016).

[67] S. Choubey, A. Ghosh, T. Ohlsson, and D. Tiwari, Neutrino physics with non-standard interactions at INO, J. High Energy Phys. 12 (2015) 126.

[68] S. P. Behera, A. Ghosh, S. Choubey, V. M. Datar, D. K. Mishra, and A. K. Mohanty, Search for the sterile neutrino mixing with the ICAL detector at INO, Eur. Phys. J. C 77, 307 (2017).

[69] B. Pontecorvo, Neutrino experiments and the problem of conservation of leptonic charge, Zh. Eksp. Teor. Fiz. 53, 1717 (1967) [Sov. Phys. JETP 26, 984 (1968)].

[70] B. Pontecorvo, Mesonium and antimesonium, Zh. Eksp. Teor. Fiz. 33, 549 (1957) [Sov. Phys. JETP 6, 429 (1957)].

[71] Z. Maki, M. Nakagawa, and S. Sakata, Remarks on the unified model of elementary particles, Prog. Theor. Phys. 28, 870 (1962).

[72] A. M. Dziewonski and D. L. Anderson, Preliminary reference Earth model, Phys. Earth Planet. Inter. 25, 297 (1981).

[73] D. Casper, The nuance neutrino physics simulation, and the future, Nucl. Phys. B, Proc. Suppl. 112, 161 (2002).

[74] T. K. Gaisser and M. Honda, Flux of atmospheric neutrinos, Annu. Rev. Nucl. Part. Sci. 52, 153 (2002).

[75] Jun Kameda, Detailed studies of neutrino oscillations with atmospheric neutrinos of wide energy range from $100 \mathrm{MeV}$ to $1000 \mathrm{GeV}$ in Super-Kamiokande, Ph.D. thesis, University of Tokyo, 2002. 
[76] M. C. Gonzalez-Garcia and M. Maltoni, Atmospheric neutrino oscillations and new physics, Phys. Rev. D 70, 033010 (2004).

[77] P. Adamson et al., Search for sterile neutrino mixing in the MINOS long-baseline experiment, Phys. Rev. D 81, 052004 (2010).
[78] J. F. Beacom and N.F. Bell, Do solar neutrinos decay?, Phys. Rev. D 65, 113009 (2002).

[79] S. Choubey et al., Effect of invisible neutrino decay on the sensitivity to mass hierarchy and the octant of $\theta_{23}-\mathrm{A}$ study with INO ICAL (to be published). 